\title{
Climate feedbacks determined using radiative kernels in a multi-thousand member ensemble of AOGCMs
}

\author{
Benjamin M. Sanderson · Karen M. Shell • \\ William Ingram
}

Received: 6 March 2009/Accepted: 1 September 2009/Published online: 17 September 2009

(C) Springer-Verlag 2009

\begin{abstract}
The use of radiative kernels to diagnose climate feedbacks is a recent development that may be applied to existing climate change simulations. We apply the radiative kernel technique to transient simulations from a multithousand member perturbed physics ensemble of coupled atmosphere-ocean general circulation models, comparing distributions of model feedbacks with those taken from the CMIP-3 multi GCM ensemble. Although the range of clear sky longwave feedbacks in the perturbed physics ensemble is similar to that seen in the multi-GCM ensemble, the kernel technique underestimates the net clear-sky feedbacks (or the radiative forcing) in some perturbed models with significantly altered humidity distributions. In addition, the compensating relationship between global mean atmospheric lapse rate feedback and water vapor feedback is found to hold in the perturbed physics ensemble, but large differences in relative humidity distributions in the ensemble prevent the compensation from holding at a regional scale. Both ensembles show a similar range of response of global mean net cloud feedback, but the mean of the perturbed physics ensemble is shifted towards more
\end{abstract}

B. M. Sanderson $(\bowtie)$

National Center for Atmospheric Research,

1850 Table Mesa Dr, Boulder, CO 80305, USA

e-mail: bsander@ucar.edu; sanderson@atm.ox.ac.uk

K. M. Shell

College of Oceanic and Atmospheric Sciences,

Oregon State University, Corvallis, USA

W. Ingram

Atmospheric, Oceanic and Planetary Physics,

University of Oxford, Oxford, UK

W. Ingram

Met Office Hadley Centre, Exeter, UK positive values such that none of the perturbed models exhibit a net negative cloud feedback. The perturbed physics ensemble contains fewer models with strong negative shortwave cloud feedbacks and has stronger compensating positive longwave feedbacks. A principal component analysis used to identify dominant modes of feedback variation reveals that the perturbed physics ensemble produces very different modes of climate response to the multi-model ensemble, suggesting that one may not be used as an analog for the other in estimates of uncertainty in future response. Whereas in the multi-model ensemble, the first order variation in cloud feedbacks shows compensation between longwave and shortwave components, in the perturbed physics ensemble the shortwave feedbacks are uncompensated, possibly explaining the larger range of climate sensitivities observed in the perturbed simulations. Regression analysis suggests that the parameters governing cloud formation, convection strength and ice fall speed are the most significant in altering climate feedbacks. Perturbations of oceanic and sulfur cycle parameters have relatively little effect on the atmospheric feedbacks diagnosed by the kernel technique.

Keywords Feedbacks - Kernel - Climate - Sensitivity · Ensemble · PPE - Perturbed physics .

Climateprediction.net · CMIP

\section{Introduction}

The temperature response of the climate system to anthropogenic greenhouse gas forcing scenarios is a critical factor in assessing the impacts of those possible future paths on society and the wider environment (Caldeira et al. 2003). This temperature response can be estimated by 
observing past changes in atmospheric forcing and the resultant change in global or regional climates (Hall and Qu 2006; Forster and Gregory 2006), or by examining the response of General Circulation Models (GCMs) in simulations of different scenarios at both global (Knutti et al. 2008; Yokohata et al. 2008) and regional scales (Murphy et al. 2007; Webb et al. 2006). The interpretation of GCM simulations is complicated when evaluating the differing responses of GCMs from the world's major climate modeling centers-raising questions both of how this spread should be represented probabilistically (Tebaldi and Knutti 2007) and how the differences in GCM response relate to feedbacks in the climate system.

\subsection{Feedback analysis in multi-GCM ensembles}

Feedbacks occur when changes in state (caused by an initial forcing) further force the system. At equilibrium, the anthropogenic forcing at the top of atmosphere $(G)$ must be balanced by changes in the outgoing longwave $(F)$ and absorbed shortwave $(Q)$ fluxes such that:

$G=\frac{\Delta(F-Q)}{\Delta T_{s}} \Delta T_{s}=\lambda \Delta T_{s}$,

where $\lambda$ is the global feedback parameter and $T_{s}$ is the global mean surface temperature, making the assumption of a linear relationship between radiative response and surface temperature.

The identification and separation of feedbacks in climate models has been identified as an essential step in understanding and constraining the future climate system response (Bony et al. 2006). In recent years, various techniques have been proposed in order to achieve this goal. The explicit separation of feedbacks and forcing in a zero-dimensional global climate model was explored in the pioneering work of Hansen et al. (1985). Separation of clear sky and cloudy sky feedbacks, however, was achieved by Cess and Potter (1988) in a simple but effective and often emulated experiment examining the response of the all sky and clear sky radiative budget to a $2 \mathrm{~K}$ perturbation in sea surface temperature. The difference between the allsky and clear sky response is then said to be the cloud radiative forcing (CRF).

Wetherald and Manabe (1988) introduced the technique later referred to as the partial radiative perturbation (PRP) technique used more recently in Colman (2003), which uses offline calculations to examine the radiative effect of individual variables (such as water vapor, cloud distribution and atmospheric temperatures) by taking the variable from the climate change simulation and substituting it into a control simulation. It was noted by Zhang et al. (1994) and Soden et al. (2004) that the CRF and PRP techniques produce different estimates for cloud feedback because the cloud feedbacks inferred by changes in CRF include some of the effects of changes in water vapor and temperature distribution in addition to changes in the cloud distribution.

In the PRP technique, an assumption of linearity allows $\lambda$ to be separated into components, such that:

$\lambda=\sum_{X} \lambda_{X}$

where $\lambda_{X}$ is the radiative response to a parameter $X$ (for example, surface albedo, atmospheric temperature or humidity):

$\lambda_{X}=-\frac{\delta(F-Q) \delta X}{\delta X \quad \delta T_{s}}$

Colman et al. (1997) point out that this assumption is incorrect if different parameters are spatially correlated (as is the case for humidity and cloud cover). This problem can be accounted for by explicitly decorrelating the result using a further independent simulation. An alternative solution proposed by Soden et al. (2008) is to consider changes in the mean state of $X$ between a control simulation and a climate change simulation and shift the distribution in the control simulation by that difference-thus preserving any correlations present between variables in the control. Perturbing the variable $X$ by a small amount $\delta X$ and observing the top of atmosphere flux change $\delta(F-Q)$ gives a radiative kernel which gives the differential response, without the computational expense of the full radiation code:

$\overline{F(X+\delta \bar{X})}-\overline{F(X)} \approx \overline{\frac{\partial F}{\partial X}(X)} \delta \bar{X}=K_{L W}^{X} \delta \bar{X}$,

$\overline{Q(X+\delta \bar{X})}-\overline{Q(X)} \approx \overline{\frac{\partial Q}{\partial X}(X)} \delta \bar{X}=K_{S W}^{X} \delta \bar{X}$,

where 4 and 5 describe the longwave and shortwave kernels $K_{L W}$ and $K_{S W}$, respectively. The kernel must have the same dimensions as the variable $X$ itself, hence if $X$ represents atmospheric temperature on model levels, then the kernel must be also be defined on model levels and must be explicitly calculated by perturbing the temperature of each model level in turn and observing the top of atmosphere response. The product of the kernel and the perturbation field $\Delta X$ gives the "field effect", which when integrated vertically gives the radiative top of atmosphere response. The methodology allows the decomposition of atmospheric response to forcing in a similar fashion to the PRP technique, but does not require each variable to be individually perturbed in each GCM analyzed-thus allowing the authors to analyze the stock simulations produced for the World Climate Research Programme's Coupled Model Inter-comparison Project Stage 3 (CMIP-3).

Soden et al. (2008) used radiative kernels to isolate different tropospheric feedbacks in the CMIP-3 ensemble, 
and stratospheric levels are not included in their integration. However, fluxes in Soden et al. (2008) are calculated at the top of the model atmosphere, thereby assuming that the net dynamical heating of the stratosphere is unchanged in the simulation, but decreasing the sensitivity of the technique to the height of the tropopause. Some earlier studies (Colman (2003; Held and Soden 2000) calculated feedbacks over the entire atmospheric column, which incorrectly diagnoses the lapse rate feedback (defined as the difference between the full atmospheric temperature feedback and the feedback assuming the surface air temperature change is constant throughout the troposphere). In this study, all feedbacks are calculated over the full atmospheric column apart from the lapse rate feedback, which is integrated over the troposphere only. The use of the full-atmosphere kernel allows the total clear-sky feedback to be verified against the GCM derived total clear-sky feedback. In the process we are including stratospheric adjustment to $\mathrm{CO}_{2}$ forcing in the feedback which is judged by Soden et al. (2008) to not be a feedback in the true sense of the atmosphere responding to the surface temperature change. However, the question is somewhat philosophical as the difference between full-column and troposphere only calculations was found to be significant only in the case of the lapse rate feedback, and the need to verify the technique in a perturbed physics environment was one of the aims of this study.

The question of whether a kernel derived in one GCM might be successfully applied to another was addressed in Soden et al. (2008). The authors used kernels derived in the NCAR and Geophysical Fluid Dynamics Laboratory (GFDL) models as well as a kernel derived from the Centre for Australian Weather and Climate Research (CAWCR) model, and applied the kernels to transient SRES A1B simulations prepared for the IPCC AR4. They found that the feedback calculations were relatively insensitive to the choice of kernel used, typically agreeing within $5 \%$. The largest differences arose in the surface albedo calculations where there was a $30 \%$ difference in magnitude between the NCAR and CAWCR kernels for the all-sky calculation.

\subsection{Feedback analysis in perturbed physics ensembles}

One aspect of the uncertainty in the GCM representation of physical processes lies in the choice of parametrization used for physical processes occurring at sub-grid scales. The values of parameters are often determined by tuning within a physically plausible range in order to best simulate past climate conditions. This is a necessary and practical step in order to create a climate model which best represents reality, but it leaves questions of how to incorporate uncertainty in GCM parameters in a future climate change scenario which, by definition, cannot be verified.
Therefore, it is of some value to study versions of the GCM where the parameters are 'de-tuned' to values that are still physically plausible but perhaps not optimal. Such an experiment produces a large number of simulations, and thus relatively few 'perturbed physics ensembles' have been created, but using those which do exist to study climate feedbacks allows the physical feedback processes to be related back to the parameter assumptions which are made during model design.

Several studies have attempted to categorize feedbacks in this perturbed physics framework; Webb et al. (2006), for example examined global and local cloud feedback patterns using an ensemble based upon a Met Office Hadley Centre Climate model, categorizing different feedbacks by the relative changes in longwave and shortwave cloud forcing and relating these to changes in cloud distribution. Other work has used statistical techniques to isolate different feedback mechanisms; Sanderson et al. (2008b) used spatial correlations derived from the climateprediction.net perturbed physics ensemble, again based upon the Met Office Hadley Centre model, to identify spatial patterns of clear sky and cloud forcing feedback that were correlated with the perturbed parameter perturbations made within the ensemble.

However, the techniques used in the studies above could provide only limited information on the physical origins of the feedback processes, mainly through inference and some knowledge of the likely effects of the perturbed parameters themselves. Both studies, and especially Webb et al. (2006), showed how much local cloud feedbacks varied within the perturbed physics ensembles compared to multiGCM ensembles such as CMIP-3. Neither study considered the extent of feedbacks associated with water vapor, lapse rate, surface temperature and albedo and how these might relate to physical parametrization.

Therein lies the focus of our study, to use the radiative kernel technique (Soden and Held 2006; Soden et al. 2008) to identify key physical feedback processes present in the climateprediction.net perturbed physics ensemble of climate models. The methodology is well suited to both the climateprediction.net and the CMIP-3 ensemble, where the data required for the PRP technique is simply unavailable.

\section{Methodology}

\subsection{The Community Atmosphere Model kernel}

The radiative kernel we utilize for this investigation was created by Shell et al. (2008) using the Community Atmosphere Model version 3 (CAM) from the National Center for Atmospheric Research (NCAR). They produced longwave kernels for surface temperature, atmospheric 
water vapor and temperature. Shortwave kernels were produced for surface albedo and atmospheric water vapor. Finally, a kernel for carbon dioxide concentration is necessary to remove the greenhouse forcing from the sum of feedbacks. Cloud feedbacks cannot be calculated directly using this technique because the relationship between the radiative effects of clouds and model cloud output diagnostics is strongly non-linear, making a (linear) cloud kernel infeasible.

Kernels are each calculated by making small perturbations and diagnosing the response at the top of the atmosphere. For the case of the atmospheric temperature kernel, each model level is raised in temperature by $1 \mathrm{~K}$, and the TOA response is observed. For the water vapor kernel, the specific humidity change is equivalent to a $1 \mathrm{~K}$ rise in temperature at constant relative humidity. Surface albedos are modified by 0.01 to yield the kernel plotted. The carbon dioxide kernel is the instantaneous longwave top of atmosphere radiative response to a doubling of carbon dioxide, so to estimate the $\mathrm{CO}_{2}$ forcing in the A1B scenario used for each of the simulations in this study we assume the forcing scales as the logarithm of the global mean atmospheric carbon dioxide concentration. We scale the forcing to be consistent with $\mathrm{A} 1 \mathrm{~B} \mathrm{CO}_{2}$ concentrations in 2075 . Forcing changes from aerosols cannot, at present, be calculated by the kernel methodology because there is no clear linear relationship between aerosol concentrations and their resultant direct and indirect radiative forcing. Thus, the adjusted CRF values shown in this study (as in previous studies using this methodology) also include forcing changes due to changes in aerosol concentrations. Further details of the issues involved with the creation of the kernels are given in Shell et al. (2008).

Shell et al. (2008) found that when the monthly calculated kernels (which we use in this study) were applied to doubled $\mathrm{CO}_{2}$ climate sensitivity experiments with the CAM, the kernels could reproduce longwave zonal modelcalculated clear-sky flux from the double- $\mathrm{CO}_{2}$ simulations within $10 \%$. Global longwave clear-sky fluxes agreed to within $25 \%$ and the shortwave kernel was accurate within $15 \%$ in the Shell et al. (2008) study. The errors for the revised kernels used in this study are somewhat more accurate, with errors of 2 and $8 \%$, respectively for longwave and shortwave clear-sky kernels (K.Shell, personal communication).

Soden et al. (2008) compared results using monthly derived kernels in the GFDL model with feedbacks derived using a de-correlated PRP approach-with temperature, water vapor and surface albedo feedbacks accurate within $2 \%$ and cloud feedbacks accurate within $10 \%$ (these errors cannot be directly compared to those found in the Shell et al. (2008), which validate kernel derived feedbacks against net GCM calculated clear-sky feedbacks). In this study we use monthly kernels because the lack of availability of daily data from the climateprediction.net ensemble.

\subsubsection{Corrected $\triangle C R F$}

Kernels are derived both for clear-sky conditions and for all-sky conditions. This approach allows for the calculation of 'corrected $\triangle \mathrm{CRF}$ ' (Soden et al. 2008). Cloud radiative forcing (CRF) is the effect of clouds in a given climate and is calculated as the difference between the clear-sky flux and the all-sky flux. Cloud feedbacks are often estimated (e.g. Cess and Potter 1988) as the difference in CRF between two climate states $(\triangle \mathrm{CRF})$. However, it has long been established (Zhang et al. 1994) that this value does not represent the full radiative effect of the cloud changes in the GCM. The value of the $\triangle \mathrm{CRF}$ may be altered by changes in specific humidity and temperature-without any change in cloud distribution. Similarly, changes in surface albedo or temperature can affect the $\triangle \mathrm{CRF}$ between two climate states. Using the difference between radiative kernels calculated for clear-sky fluxes and all-sky fluxes, it is possible to calculate the component of $\triangle \mathrm{CRF}$ which are due to changes in $\mathrm{CO}_{2}$ concentrations, atmospheric temperature, humidity and surface albedo. The $\Delta$ CRF between two climate states can then be easily separated into a component due to non-cloud variables with a residual due to changes in cloud distribution, henceforth referred to as the "corrected $\triangle \mathrm{CRF}$ ".

\subsection{The climateprediction.net transient ensemble}

The climateprediction.net experiment (Stainforth et al. 2005) is a multi-thousand member perturbed physics ensemble of experiments conducted using the otherwise idle processing time on personal computers donated by interested volunteers. The simulations described in Stainforth et al. (2005) were mixed-layer models used to determine the climate sensitivity to a doubling of carbon dioxide. The simulations we use here, however, are taken from the next stage of the experiment which use a fully coupled dynamical ocean and simulate transient climate behavior. The GCM is a low ocean resolution version of the 3rd Met Office Hadley Centre model (HadCM3L) using a resolution of $2.5^{\circ}$ in latitude and $3.75^{\circ}$ in longitude (for both atmosphere and ocean), with 19 atmospheric levels. Further details of the climateprediction.net experimental design are given in the Appendix "Model setup".

The parameters perturbed in the experiment relate to the atmosphere, the sulfur cycle and the ocean model. Brief explanations of the parameters perturbed in this experiment are given in Table 4. Further information on the perturbed parameters may be found in Murphy et al. (2004), or in the 
Met Office technical notes. One limitation of the public nature of the experiment is that it is impossible to return all model output for analysis.

The variables are returned as zonally averaged fields on model levels. Thus, this study must test the ability of a zonally averaged kernel used with the data to approximate the fully gridded calculation described in Soden et al. (2008) and Shell et al. (2008). Thus, we also repeat those calculations of Soden et al. (2008) computed with the NCAR kernel and the IPCC AR-4 simulations-and compare those results to a zonally averaged approximation. The zonally averaged kernels are computed from those described in Shell et al. (2008) and are plotted in Fig. 1. Results presented in Sect. 3 suggest that the zonally averaged kernels are an acceptable substitute for the fully gridded kernels.

Other issues with the use of the climateprediction.net dataset lie in the absence of certain surface fields from the dataset. The first of these is the surface temperature (only
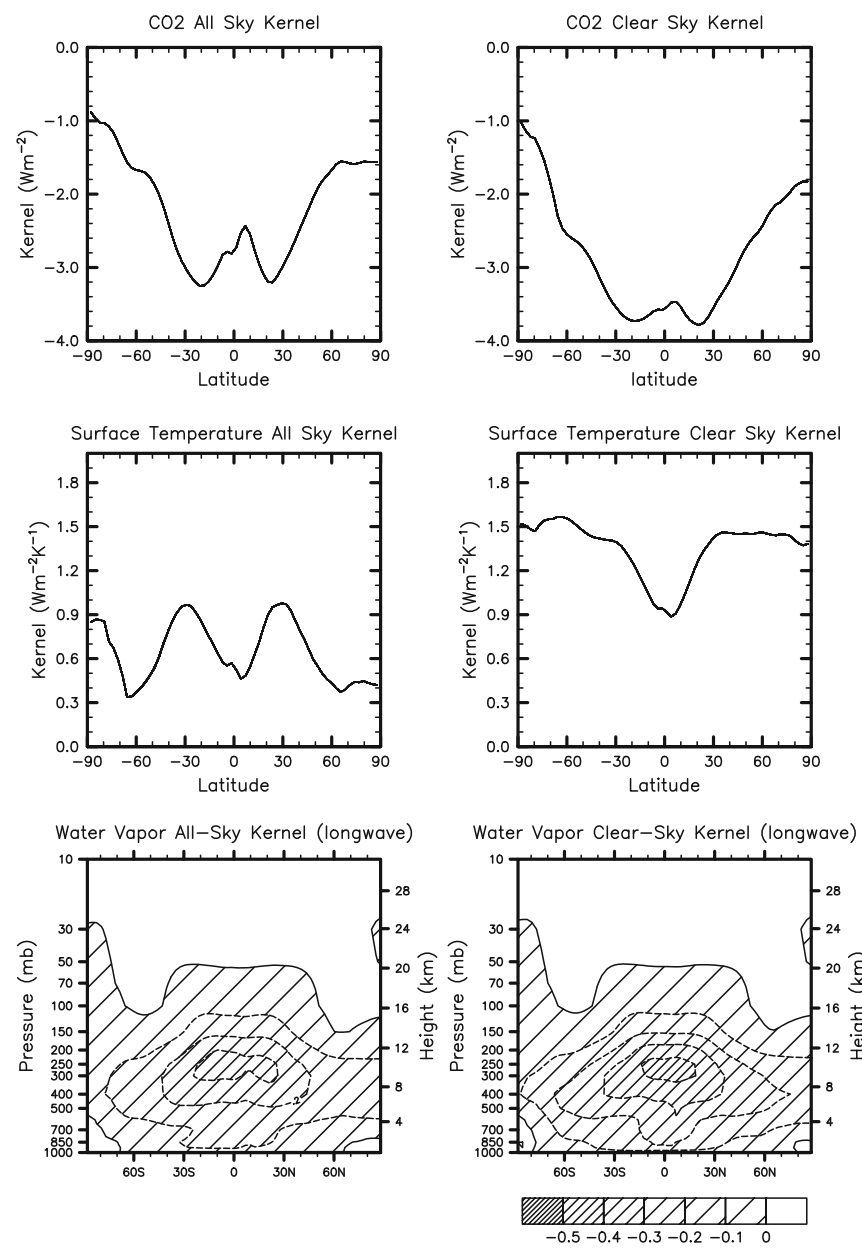

Fig. 1 Zonally averaged clear-sky and all-sky radiative kernels from Shell et al. (2008) for carbon dioxide (top left, longwave only, $\mathrm{Wm}^{-2}$ ), Surface Albedo (top right, shortwave, $\mathrm{Wm}^{-2}$ ), Surface Temperature (middle left, longwave, $\mathrm{Wm}^{-2} \mathrm{~K}^{-1}$ ), Water vapor
$2 \mathrm{~m}$ air temperature is retained). Although not an ideal solution, we use the same surface temperature feedback (from the default HadCM3 experiment, which has unperturbed physics parameters) for all the climateprediction.net models. This is unlikely to have a significant effect on the net global feedback - the surface temperature feedback in the GCMs presented in Soden et al. (2008) was shown to vary by only $0.1 \mathrm{Wm}^{-2} \mathrm{~K}^{-1}$ amongst the CMIP-3 models, thus accounting for less than $5 \%$ of the variance in overall feedback. In addition, because the land surface and boundary layer schemes are not perturbed in the climateprediction.net experiment, there is no reason, per se, to expect it to vary significantly from the unperturbed model.

The other missing fields in the climateprediction.net zonally averaged fields are the surface shortwave fluxes, making a calculation of surface albedo impossible. However, the total clear-sky shortwave top of atmosphere transient change is known, so we resolve this issue in the
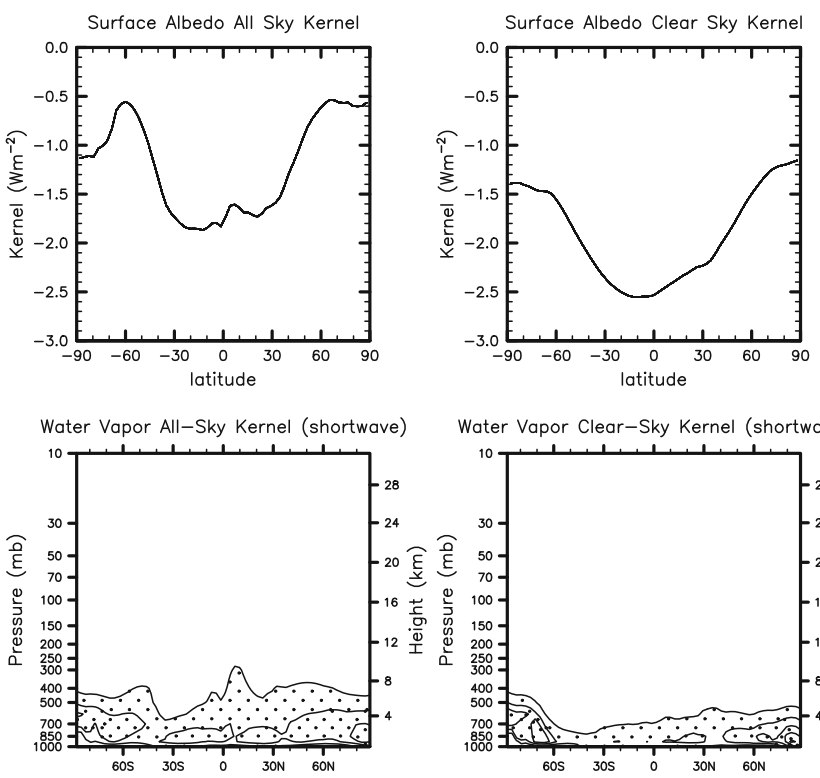

Water Vapor Clear-Sky Kernel (shortwave
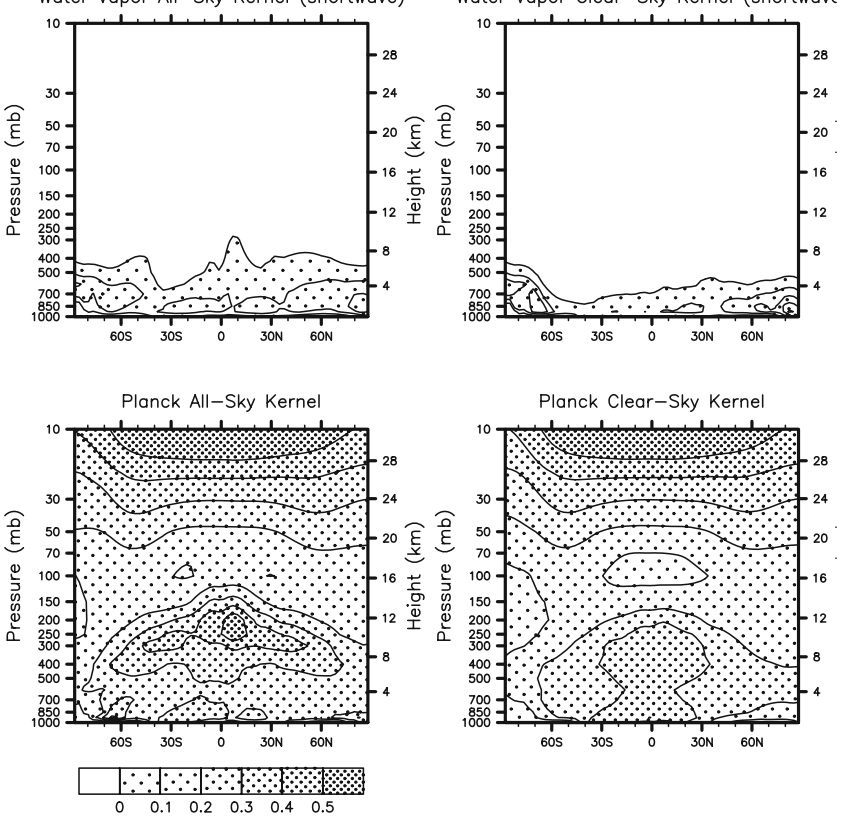

(middle right shortwave and bottom left longwave, $\mathrm{Wm}^{-2} / 100 \mathrm{hPa}$ per humidity increase corresponding to $1 \mathrm{~K}$ increase with $\mathrm{RH}$ constant) and Atmospheric temperature (Bottom right, longwave, $\mathrm{Wm}^{-2} \mathrm{~K}^{-1} / 100 \mathrm{hPa}$ ) 
clear-sky case by assuming that the albedo feedback accounts for the residual clear-sky feedback after the effect of water vapor in the shortwave energy budget has been taken into account. The disadvantage of this approach is that it does not allow the kernel calculation to be verified in the clear-sky shortwave case. The calculation of the all-sky albedo feedback is, by necessity, more contrived. The cloud shortwave corrected $\triangle \mathrm{CRF}$ requires prior knowledge of the all-sky albedo feedback, and thus the albedo feedback cannot be calculated as a residual as it was for the clear-sky case. The solution chosen was to use a "twoway" kernel calculation in order to obtain the surface albedo change from the known clear-sky flux change due to the change in surface albedo. This can be calculated by taking the full clear-sky shortwave flux change, and subtracting the component due to changes in water vapor using the shortwave clear-sky water vapor kernel.

$\Delta \alpha \approx \frac{\Delta Q(\alpha(C S))}{K_{S W}^{\alpha(C S)}+a}$,

where $\alpha$ is the surface albedo, $\Delta Q(\alpha(C S))$ is the change in shortwave clear-sky top of atmosphere flux due to changes in surface albedo and $K_{S W}^{\alpha(C S)}$ is the shortwave clear-sky surface albedo kernel. A small $\left(a=0.001 \mathrm{Wm}^{-2}\right)$ value is necessary in the denominator for the stability of the calculation in regions where the kernel $K_{S W}^{\alpha(C S)}$ was negligible. This estimate for the surface albedo change may then be used with the all-sky kernel to estimate the all-sky surface albedo feedback strength.

\subsection{Feedback analysis}

The methodology associated with the use of the kernels to identify GCM feedbacks is almost identical to that of Soden et al. (2008), but zonally averaged kernels are used in place of the full kernels. For each GCM, the difference is taken in each variable $X$ between the 1990-2000 and the 2070-2080 mean in the A1B scenario (note the latter window differs from Soden et al. (2008), who used 21002110 , as the climateprediction.net simulations only run up to 2080). The climateprediction.net data must first be linearly interpolated onto the CAM kernel horizontal resolution (2.5 latitude) and model output levels. For each variable, the resultant $\Delta X$ is multiplied by the kernel and divided by the global average temperature change to yield the field effect, which is of the same dimensions as the variable $X$. If $X$ is defined on pressure levels, then each level is weighted by the pressure difference between the top and bottom of the level, and then the feedbacks are summed vertically to yield the zonally averaged feedback profile for that variable. Feedbacks are calculated for each month, using monthly averaged kernels together with monthly averaged data for $X$. The results presented in this paper are annual mean feedbacks which average the monthly means for each variable.

The lapse rate feedback is calculated only in the troposphere by taking the true atmospheric temperature feedback in the troposphere and subtracting the feedback corresponding to a uniform temperature increase in the troposphere equal to that of the surface.

\subsection{Principal component analysis}

Information on how different types of feedback are correlated, both in perturbed physics and in multi-GCM ensembles, could potentially provide better constraints on overall climate sensitivity. However correlations of global mean feedback quantities are of limited use, as any regional or multi-variable information is lost. In order to extract more meaning from large ensembles such as climateprediction.net, it has proved useful in the past to use principal component analysis (PCA) to determine major modes of variation within the ensemble (Sanderson et al. 2008b; Sanderson et al. 2008a).

First, each of the all-sky feedbacks are area-weighted. The arrays for each feedback type are then concatenated (where the variables are albedo (shortwave), water vapor and cloud (both longwave and shortwave feedbacks) and lapse rate and surface temperature (longwave). Surface temperature feedbacks are considered only in the CMIP-3 case, because the default HadCM3 value was assumed for all climateprediction.net models. The ensemble mean is subtracted from each ensemble member and the resulting matrix is of dimensions $\left(n_{v} * n_{\text {lat }}\right)$ by $N$, where $n_{v}$ is the number of feedback variables $\left(n_{v}=7\right.$ for CMIP-3 and 6 for climateprediction.net due to the exclusion of surface temperature feedback), $n_{\text {lat }}$ is the number of latitudinal coordinates $\left(n_{\text {lat }}=72\right)$ and $N$ is the number of members of the ensemble used $(N=1,615$ for climateprediction.net and $N=18$ for CMIP-3). This matrix is then subjected to PCA, such that the leading modes represent the largest ensemble variance.

The result of the principal component analysis is a set of empirical orthogonal functions (EOFs), principal components and eigenvalues. The EOFs are concatenated zonal mean feedback patterns $E_{j x}$, where $j$ is the EOF number and x is the feedback dimension. The principal components $P_{i j}$ are the coefficients of the EOF $j$ in each ensemble member $i$. The eigenvalues $\lambda_{j}$ are the variance represented by the EOF $j$. We truncate the EOF set so that $95 \%$ of the ensemble variance is retained (requiring 12 modes), but the results of the parameter regression discussed below are insensitive to any increase in truncation length beyond 10 modes, because the calculation weights each mode by its eigenvalue making high order modes insignificant. 
Table 1 Base climate fields

\begin{tabular}{lll}
\hline Short name & Model name & $\begin{array}{l}\text { Atmos } \\
\text { resolution }\end{array}$ \\
\hline ncar_ccsm3_0 & NCAR CCSM 3.0 & $T 85$ \\
ncar_pcm1 & NCAR PCM1 & $T 42$ \\
giss_model_e_h & ModelE20/HYCOM 4x5xL20 & $4^{\circ} \times 5^{\circ}$ \\
giss_model_e_r & ModelE20/Russell 4x5xL20 & $4^{\circ} \times 5^{\circ}$ \\
csiro_mk3_0 & CSIRO mark 3.0 & $T 63$ \\
cccma_cgcm3_1 & CCCMA CGCM 3.1 T47 & $T 47$ \\
cccma_cgcm3_1 & CCCMA CGCM 3.1 T63 & $T 63$ \\
cnrm_cm3 & CNRM-CM3 & $T 63$ \\
mpi_echam5 & MPI Echam 5.0 & $T 63$ \\
iap_fgoals1_0_g & IAP/LASG FGOALS 1.0g & $2.8^{\circ} \times 2.8^{\circ}$ \\
gfdl_cm2_0 & GFDL CM2.0 & $2.5^{\circ} \times 2.0^{\circ}$ \\
gfdl_cm2_1 & GFDL CM2.1 & $2.5^{\circ} \times 2.0^{\circ}$ \\
inmcm3_0 & INM-CM3.0 & $5.0^{\circ} \times 4.0^{\circ}$ \\
ipsl_cm4 & IPSL CM4.0 & $2.5^{\circ} \times 3.75^{\circ}$ \\
miroc3_2_hires & Miroc 3.2 (hires) & $T 106$ \\
miroc3_2_medres & Miroc 3.2 (medres) & $T 42$ \\
mri_cgcm2_3_2a & MRI CGCM2.3.2a & $2.5^{\circ} \times 2.0^{\circ}$ \\
ukmo_hadgem1 & MO HadGEM1 & $1.25^{\circ} \times 1.875^{\circ}$ \\
\hline CMP3 &
\end{tabular}

CMIP-3 models used in the analysis, together with the original GCM resolutions

This process can then be repeated with the CMIP-3 ensemble to establish whether leading spatial correlations between different feedback types are consistent between the perturbed physics and inter-GCM ensembles. In this case, the only difference is that $N$ is now the number of suitable members of CMIP-3. $N$ is 18 , listed in Table 1 showing those models with sufficient output variables in the A1-B scenario to compute the kernel feedback values.

\subsection{Parameter regression}

One of the most useful aspects of a performing a feedback analysis on a perturbed physics ensemble lies in the ability to relate physical parametrizations to feedback processes in the perturbed physics framework. The simplest way to examine these relationships is to consider the cross-correlations $C_{n}^{X}$ between the set of perturbed parameter values and different kernel-derived global mean feedbacks. Thus, those parameters with a positive or negative correlation to global mean feedbacks in the ensemble may be identified:

$C_{n}^{X}=\sum_{i=1}^{N} \frac{\left(B^{X}(i)-\overline{B^{X}}\right) \times\left(P_{n}(i)-\overline{P_{n}}\right)}{\sigma\left(B^{X}\right) \sigma\left(P_{n}\right)}$,

where $i$ is the ensemble member out of a possible $N$, where $N=1,615$ in this case (a fully sampled parameter space would yield nearly $8.8 \times 10^{13}$ simulations for this parameter set, but thankfully we only require a range of behavior for this study, rather than an exhaustive parameter sweep), $B^{X}$ is the global mean kernel-derived feedback corresponding to the variable $X, P_{n}(i)$ is the value of the $n$th model parameter in simulation $i$, over-bars indicate an average across all ensemble members, and $\sigma$ is the standard deviation of the feedback or parameter value.

To further understand the correlated feedback processes in the perturbed physics ensemble, it is also of some use to consider the original parameter perturbations and how they influence the feedbacks. One way to achieve this is to use a linear regression to project from the parameter space onto to a truncated set of EOFs, in a technique first proposed in Sanderson et al. (2008b). This, in practice involves creating a parameter matrix $p_{i l}$, where $i$ is the ensemble member and $l$ is the parameter number from a total of 24 independent parameters. The parameter matrix is standardized such that the mean of each column is zero (i.e., for each parameter, the mean parameter value for the ensemble is zero.) and the standard deviation is one. A simple regression equation can then be used to express each the principal components $P_{i j}$ as a function of the parameter values.

$P_{i j}=\sum_{l=1}^{24} p_{i l} \beta_{l j}+$ noise.

We solve for $\beta_{l j}$ using a standard ordinary least squares approach. Once known, $\beta_{i j}$ may be used to reconstruct a feedback pattern $E_{l s}$ (for each parameter $l$ over the feedback/spatial domain $s$ ) which is expected from a positive unit change in each normalized parameter (the actual parameter perturbation to which this corresponds is dependent on the original climateprediction.net sampling strategy and will be on the order of the perturbations listed in 4).

$E_{l s}=\sum_{j=1}^{T} \beta_{l j} \lambda_{j} E_{j s}$,

gives some indication of the zonal pattern of each type of feedback associated with a perturbation of the model parameter $l$. Of course, such an analysis will not capture any non-linearity that might be associated with multiple parameter perturbations. To extract such effects, a nonlinear analysis is required. For example, Sanderson et al. (2008a) used a neural network to model the parameter dependence of climate sensitivity in a non-linear framework. However, the use of such techniques introduces additional complexities and uncertainties in the training and fitting process which were deemed an unnecessary complication in this case, where a first order estimate of the parameter effects on climate feedbacks is desired. 


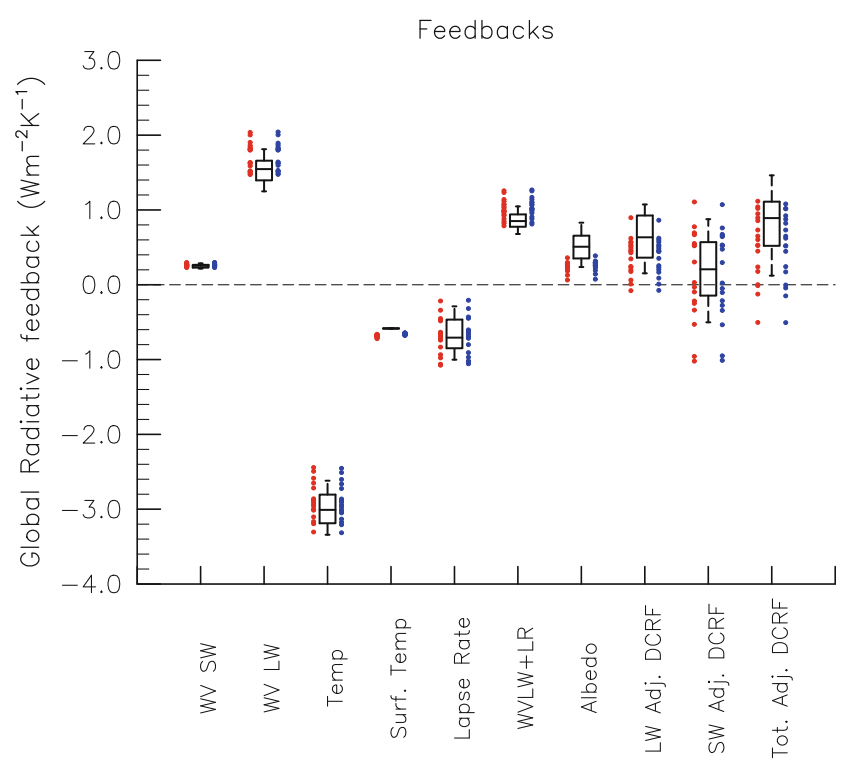

Fig. 2 A comparison of global mean feedback values for different variables. Variables represent Longwave and Shortwave Water Vapor, Atmospheric Temperature, Surface Temperature, Lapse Rate, Albedo, Lapse Rate plus Water Vapor Longwave, Longwave and Shortwave Adjusted $\triangle \mathrm{CRF}$ and Total Adjusted $\Delta \mathrm{CRF}$. Red dots represent model feedbacks for the CMIP-3 ensemble computed with the full, gridded radiative kernel. Blue dots represent the zonally averaged kernel approximation for the same GCMs. Box plots show the distribution of the climate prediction.net transient simulations, where the 1,10,50,90 and 99th percentiles are shown by the boxes and whiskers. Sign convention is such that positive numbers indicate positive feedbacks, which reduce outgoing radiation at the top of the model atmosphere with increasing surface temperature

\section{Results}

\subsection{Verification of zonally averaged approximation}

Before the distributions of feedbacks in climateprediction.net may be compared to that in the CMIP-3 ensemble, it is necessary to verify that the zonally averaged kernels can reproduce similar values to the fully gridded kernels used in Soden et al. (2008). Figure 2 demonstrates the distribution of CMIP-3 kernel derived feedbacks using both approaches, and both results are presented in Tables 2 and 3. We find that, in general, the zonally averaged kernel is an acceptable approximation to the full gridded calculations, with a mean error of less than $0.1 \mathrm{Wm}^{-2} \mathrm{~K}^{-1}$ (see Tables 3, 4).

\subsection{The climateprediction.net feedback distribution}

The distribution of the models in the climateprediction.net ensemble can thus be compared with that of the CMIP-3 ensemble. We find a similar range of longwave non-cloud feedbacks in the CMIP-3 ensemble and the climateprediction.net ensemble. Figure 2 shows that the longwave water vapor feedback varies in both ensembles by $0.6 \mathrm{Wm}^{-2} \mathrm{~K}^{-1}$. Atmospheric temperature feedbacks vary in the CMIP-3 ensemble by more than $1.0 \mathrm{Wm}^{-2} \mathrm{~K}^{-1}$, while the climateprediction.net distribution varies by $0.8 \mathrm{Wm}^{-2} \mathrm{~K}^{-1}$. The apparent spread of feedback strength in the climateprediction.net ensemble may, however be an underestimate, as discussed in Sect. 3.3.
Table 2 Zonally averaged kernel feedbacks

CMIP-3 feedback values in $\mathrm{Wm}^{-2} \mathrm{~K}^{-1}$ using the zonal mean kernel

Columns correspond to Albedo (A), Water vapor Longwave (WL), Water vapor Shortwave (WS), Surface Temperature (T*), Atmospheric Temperature (TA), Lapse Rate (L), Longwave Adjusted Cloud Feedback (CL) and Shortwave Adjusted Cloud Feedback (CS)

\begin{tabular}{llllllllr}
\hline Model & A & WL & WS & T* & TA & L & CL & CS \\
\hline ncar_ccsm3_0 & 0.29 & 1.51 & 0.24 & -0.66 & -2.62 & -0.45 & 0.23 & -0.28 \\
ncar_pcm1 & 0.29 & 1.52 & 0.24 & -0.65 & -2.56 & -0.43 & 0.44 & -0.95 \\
giss_model_e_h & 0.07 & 1.86 & 0.27 & -0.68 & -3.11 & -1.06 & 0.62 & -0.10 \\
giss_model_e_r & 0.14 & 1.80 & 0.26 & -0.67 & -3.02 & -0.97 & 0.57 & -0.05 \\
csiro_mk3_0 & 0.26 & 1.62 & 0.25 & -0.65 & -2.69 & -0.61 & -0.08 & 0.52 \\
cccma_cgcm3_1 & 0.29 & 2.04 & 0.29 & -0.67 & -3.27 & -1.04 & 0.48 & 0.53 \\
cccma_cgcm3_1 & 0.39 & 1.83 & 0.27 & -0.65 & -2.98 & -0.70 & 0.44 & 0.47 \\
cnrm_cm3 & 0.27 & 1.81 & 0.26 & -0.66 & -2.96 & -0.80 & 0.08 & 0.64 \\
mpi_echam5 & 0.26 & 1.81 & 0.26 & -0.65 & -3.17 & -0.91 & 0.35 & 0.30 \\
iap_fgoals1_0_g & 0.32 & 1.47 & 0.24 & -0.65 & -2.41 & -0.21 & 0.45 & -0.21 \\
gfdl_cm2_0 & 0.30 & 1.80 & 0.27 & -0.66 & -2.93 & -0.70 & 0.52 & -0.54 \\
gfdl_cm2_1 & 0.19 & 2.00 & 0.29 & -0.67 & -3.14 & -1.02 & 0.86 & -1.01 \\
inmcm3_0 & 0.29 & 1.48 & 0.23 & -0.64 & -2.47 & -0.32 & 0.51 & -0.34 \\
ipsl_cm4 & 0.25 & 1.64 & 0.25 & -0.67 & -2.86 & -0.63 & 0.00 & 1.07 \\
miroc3_2_hires & 0.30 & 1.62 & 0.26 & -0.67 & -3.21 & -0.67 & 0.25 & 0.76 \\
miroc3_2_medres & 0.26 & 1.59 & 0.26 & -0.67 & -3.26 & -0.72 & 0.20 & 0.67 \\
mri_cgcm2_3_2a & 0.23 & 1.89 & 0.29 & -0.65 & -3.20 & -0.64 & 0.59 & 0.02 \\
ukmo_hadgem1 & 0.25 & 1.51 & 0.24 & -0.65 & -2.96 & -0.45 & 0.16 & 0.65 \\
\hline
\end{tabular}


Table 3 Full kernel CMIP-3 feedbacks

As for Table 2, but using the full gridded kernel

Bottom row (std_diff)shows the standard deviation, in $\mathrm{Wm}^{-2} \mathrm{~K}^{-1}$ of the difference between the zonally averaged kernel and the full kernel

Table 4 Definition of perturbed parameters as used in the subset of climateprediction.net experiments used in this analysis a Parameters are perturbed together

b Parameters are actually defined on 19 model levels, with the mean over model levels shown here

Values column indicates the possible parameter values used in the experiment for each parameter, with units (where applicable) shown in parenthesis

\begin{tabular}{lllllllll}
\hline Model & A & WL & WS & \multicolumn{1}{l}{ T* } & TA & L & CL & CS \\
\hline ncar_ccsm3_0 & 0.28 & 1.51 & 0.24 & -0.68 & -2.65 & -0.47 & 0.24 & -0.25 \\
ncar_pcm1 & 0.28 & 1.52 & 0.24 & -0.67 & -2.59 & -0.45 & 0.45 & -0.96 \\
giss_model_e_h & 0.06 & 1.86 & 0.27 & -0.72 & -3.10 & -1.08 & 0.62 & -0.10 \\
giss_model_e_r & 0.13 & 1.80 & 0.25 & -0.71 & -3.01 & -0.98 & 0.56 & -0.03 \\
csiro_mk3_0 & 0.23 & 1.63 & 0.25 & -0.68 & -2.72 & -0.63 & -0.08 & 0.53 \\
cccma_cgcm3_1 & 0.27 & 2.04 & 0.30 & -0.69 & -3.31 & -1.07 & 0.49 & 0.55 \\
cccma_cgcm3_1 & 0.36 & 1.84 & 0.27 & -0.67 & -3.01 & -0.72 & 0.43 & 0.52 \\
cnrm_cm3 & 0.27 & 1.81 & 0.26 & -0.68 & -3.00 & -0.83 & 0.06 & 0.67 \\
mpi_echam5 & 0.24 & 1.82 & 0.26 & -0.67 & -3.19 & -0.94 & 0.35 & 0.30 \\
iap_fgoals1_0_g & 0.29 & 1.47 & 0.25 & -0.67 & -2.44 & -0.22 & 0.46 & -0.22 \\
gfdl_cm2_0 & 0.29 & 1.81 & 0.27 & -0.69 & -2.96 & -0.73 & 0.53 & -0.53 \\
gfdl_cm2_1 & 0.18 & 2.00 & 0.29 & -0.71 & -3.17 & -1.06 & 0.90 & -1.02 \\
inmcm3_0 & 0.28 & 1.48 & 0.23 & -0.67 & -2.49 & -0.34 & 0.52 & -0.34 \\
ipsl_cm4 & 0.24 & 1.63 & 0.25 & -0.69 & -2.89 & -0.65 & 0.01 & 1.11 \\
miroc3_2_hires & 0.29 & 1.62 & 0.26 & -0.68 & -2.94 & -0.70 & 0.24 & 0.78 \\
miroc3_2_medres & 0.25 & 1.59 & 0.27 & -0.68 & -2.88 & -0.74 & 0.20 & 0.69 \\
mri_cgcm2_3_2a & 0.21 & 1.90 & 0.29 & -0.67 & -2.91 & -0.67 & 0.57 & 0.03 \\
ukmo_hadgem1 & 0.23 & 1.51 & 0.25 & -0.66 & -2.86 & -0.48 & 0.18 & 0.68 \\
std_diff & 0.011 & 0.008 & 0.005 & 0.010 & 0.014 & 0.010 & 0.015 & 0.013 \\
\hline
\end{tabular}

\begin{tabular}{|c|c|c|}
\hline Label & Description & Values \\
\hline CW_SEA & Threshold for precip. over sea ${ }^{\mathrm{a}}\left(\mathrm{kg} \mathrm{m}^{-3}\right)$ & {$[50,5,2] \times 10^{-5}$} \\
\hline CW_LAND & Threshold for precip. over land ${ }^{\mathrm{a}}\left(\mathrm{kg} \mathrm{m}^{-3}\right)$ & {$[20,2,1] \times 10^{-4}$} \\
\hline ENTCOEF & Entrainment coefficient & {$[0.6,1.0,3.0,9.0]$} \\
\hline RHcrit & Critical relative humidity & {$[0.65,0.73,0.90]^{\mathrm{b}}$} \\
\hline CT & Accretion constant $\left(\mathrm{s}^{-1}\right)$ & {$[40,10,5] \times 10^{-5}$} \\
\hline EACF & Empirically adjusted cloud fraction & {$[0.5,0.63,0.69]^{\mathrm{b}}$} \\
\hline VF1 & Ice fall speed $\left(\mathrm{m} \mathrm{s}^{-2}\right)$ & {$[0.5,1,2]$} \\
\hline DTHETA & Initial condition ensemble generator $(\mathrm{K})$ & {$[0,1, \ldots, 9] \times 10^{-2}$} \\
\hline ALPHAM & Albedo at melting point of ice & {$[0.5,0.57,0.65]$} \\
\hline DTICE & Temperature range of ice albedo variation & {$[2,5,10]$} \\
\hline I_CNV_ICE_LW & Convective cloud ice crystal type $(\mathrm{LW})^{\mathrm{a}}$ & {$[1,7]$} \\
\hline I_CNV_ICE_SW & Convective cloud ice crystal type $(\mathrm{SW})^{\mathrm{a}}$ & {$[3,7]$} \\
\hline I_ST_ICE_LW & Stratiform cloud ice crystal type $(\mathrm{LW})^{\mathrm{a}}$ & {$[1,7]$} \\
\hline I_ST_ICE_SW & Stratiform cloud ice crystal type $(\mathrm{SW})^{\mathrm{a}}$ & {$[2,7]$} \\
\hline ICE_SIZE & Ice crystal size in radiation (m) & {$[2.5,3,4] \times 10^{-5}$} \\
\hline ANTHSCA & Scaling factor for anthropogenic sulphates & {$[0.5,0.8,1.0,1.2,1.5]$} \\
\hline CLOUDTAU & Residence time of air parcel in cloud (s) & {$[3.6,10.8,32.4] \times 10^{3}$} \\
\hline LO & Sulphate mass scavenging parameter $\left(\mathrm{s}^{-1}\right)$ & {$[2.17,6.5,19.5] \times 10^{-5}$} \\
\hline NUM_STAR & Condensation threshold for accumulation & {$[1,10,100] \times 10^{5}$} \\
\hline SO2_HIGH_LEVEL & Model level for $\mathrm{SO}_{2}$ (high level) emissions & {$[1,3,5]$} \\
\hline VOLSCA & Scaling factor for volcanic emissions & {$[1,2,3]$} \\
\hline HANEY & Haney Coefficient $\left(\mathrm{s}^{-1}\right)$ & {$[163.8,81.9]$} \\
\hline HANEYSFACT & Haney Salinity Factor & {$[0.25,1.0]$} \\
\hline ISOPYC & Isopycnal diffusivity $\left(\mathrm{m}^{2} \mathrm{~s}\right)$ & {$[.2,1,2] \times 10^{3}$} \\
\hline MLLAM & Wind mixing energy scaling factor $\left(\mathrm{m}^{2} \mathrm{~s}\right)$ & {$[.2,1,2] \times 10^{3}$} \\
\hline VDIFFSURF & Background vertical tracer mixing & {$[5,10,20] \times 10^{-6}$} \\
\hline VERTVISC & Background vertical momentum mixing & {$[5,10] \times 10^{-6}$} \\
\hline
\end{tabular}


The apparent bias in values for the albedo feedback must be considered in light of the approximate method used to estimate the climateprediction.net albedo change (see Sect. 2.2). Longwave cloud feedbacks show a similar range in both CMIP-3 and climateprediction.net, but the climateprediction.net ensemble shows a shift in the distribution towards more positive feedbacks such that none of the models in the climateprediction. net show a net negative longwave cloud feedback. The range of shortwave cloud feedbacks in climateprediction.net is less than that in CMIP-3, such that the CMIP-3 ensemble shows both more positive and more negative values of global mean shortwave cloud feedback. When the total cloud feedback is considered, approximately $10 \%$ of the models show a greater positive net cloud feedback than any model in the CMIP-3 ensemble, but no models show a net negative cloud feedback in response to surface warming (whereas there are 3 models in the CMIP-3 ensemble with a net negative global mean cloud feedback).

\subsection{Feedback relationships}

Soden et al. (2008) proposed various relationships between feedback quantities, and we investigate these and others to test how robust these relationships might be in the perturbed physics environment. We would like to verify that the sum of the kernel derived feedbacks is a reasonable estimate of the true GCM evaluated feedback response. Unfortunately, it is only possible to achieve this in the clear sky, longwave case. The all-sky results use the model evaluated change in cloud forcing to estimate the cloud feedback, making it impossible to verify the accuracy of the all-sky kernels. Similarly, because of absent fields in the climateprediction.net dataset, the clear sky albedo feedback is calculated as a residual, making verification in the shortwave impossible also.

The kernel derived sum of longwave clear-sky feedbacks is shown plotted against model derived values in Fig. 3a and shows that the feedbacks in the climateprediction.net
Fig. 3 Scatter plots showing the relationship between various global mean feedback quantities in both climateprediction.net and the CMIP-3 ensemble. Black points represent members of the CMIP- 3 ensemble, while colored points are members of the climateprediction.net ensemble. Coloring is indicative of the value of the 'Entrainment Coefficient' parameter in the climateprediction.net parameter sampling scheme. 'GM' refers to global mean values, while 'CRF' refers to cloud radiative forcing

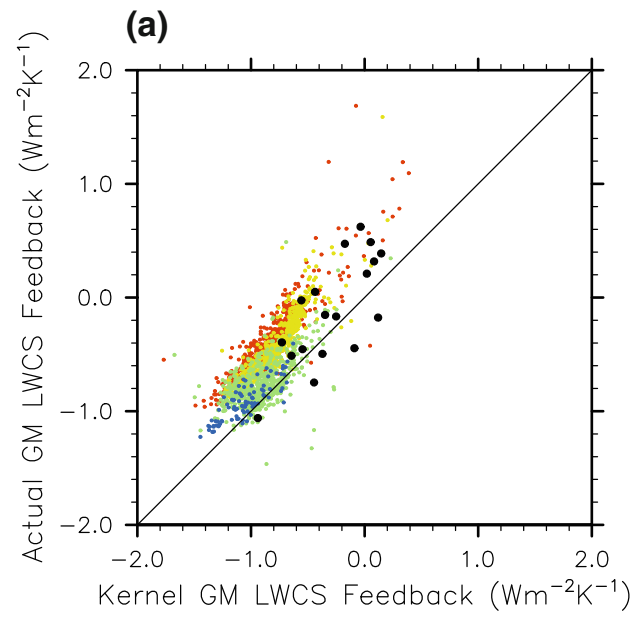

(b)

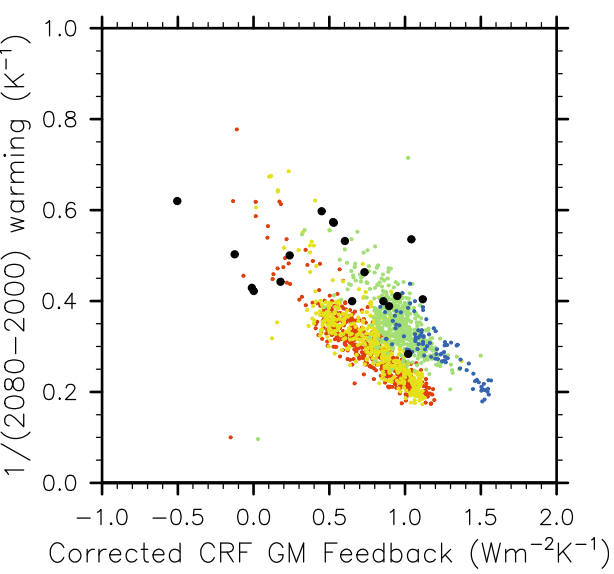

(c)

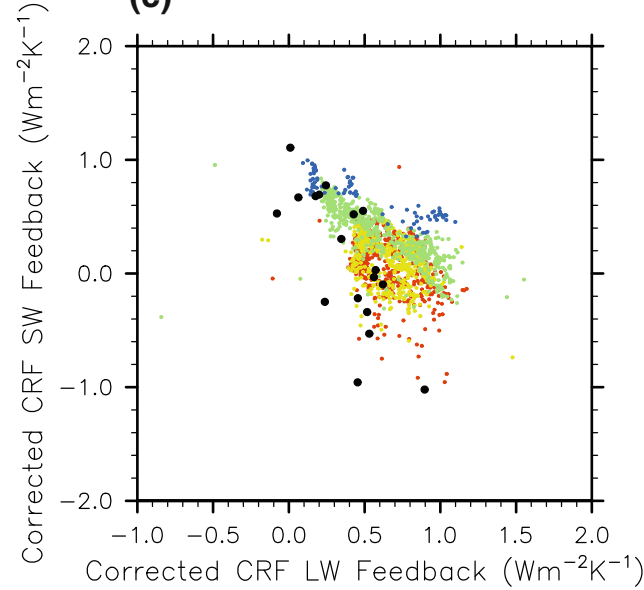

(d)

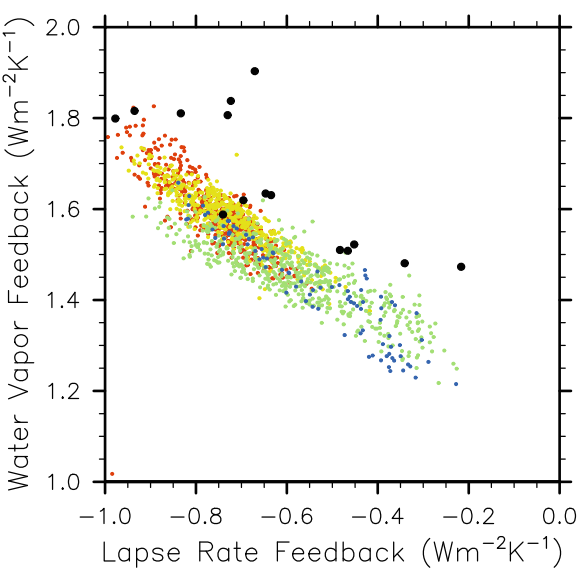

- CMIP

- entcoef $=9.0$

- entcoef $=3.0$

entcoef $=1.0$

- entcoef $=0.6$ 
ensemble are underestimated by the kernel technique by over $1.5 \mathrm{Wm}^{-2} \mathrm{~K}^{-1}$ in some cases. For the purposes of Fig. 3, we have chosen to include the value of one of the parameters, the entrainment coefficient (ENTCOEF). In this case, we find that the kernel estimate for the total feedback is more accurate for models with the standard or high value of ENTCOEF than for those models with a reduced value. This bias indicates that the CAM derived kernel is incorrectly estimating the top of atmosphere clearsky fluxes in some of the perturbed models. However, without performing a PRP experiment within one of the perturbed models, we cannot say whether the bias originates in the $\mathrm{CO}_{2}$ forcing or the feedbacks. It seems likely, however, that the forcing in models with highly perturbed humidity and temperature distributions may differ significantly from the kernel-derived estimates. Collins et al. (2006) found that even within the IPCC AR-4 models (which are arguably tuned to reproduce the observed climate), the forcing due to a doubling of carbon dioxide varied by almost a factor of 2, making it likely that the forcing in the perturbed physics environment will vary even more.

Figure $3 \mathrm{~b}$ shows the corrected change in cloud radiative forcing per unit surface temperature rise plotted against the inverse of 2080-2000 global mean temperature change (which includes all net feedbacks in the system, as well as the effects of ocean heat uptake). The corrected $\triangle \mathrm{CRF}$, as explained in Sect. 2.3, excludes changes in $\triangle \mathrm{CRF}$ which are due to changes in temperature, humidity or carbon dioxide concentrations. In the climateprediction.net models, there is an inverse relationship between corrected $\Delta \mathrm{CRF}$ and $\Delta T_{\text {global }}$ but again, this relationship is dependent on the value of ENTCOEF, indicating again that ENTCOEF has a large effect on the clear-sky component of the total feedback. However, for any given value of ENTCOEF, it is apparent that the 2080 warming in the climateprediction.net ensemble is strongly correlated with the net cloud feedback in the system, which is itself dependent on other model parameters (see Sect. 3.5).

Figure $3 \mathrm{c}$ shows a compensating relationship between longwave and shortwave cloud feedbacks in both CMIP-3 and climateprediction.net. This is to be expected from at least high level cloud changes, where increases in high cloudiness on warming would produce a negative shortwave feedback coupled with a positive longwave feedback. The longwave compensation amongst models is slightly stronger in the climateprediction.net ensemble-almost 0.7 $\mathrm{Wm}^{-2} \mathrm{~K}^{-1}$ increase in positive longwave cloud feedback for every $1 \mathrm{Wm}^{-2} \mathrm{~K}^{-1}$ increase in negative shortwave cloud feedback in contrast to CMIP-3 where there is, on average, $0.5 \mathrm{Wm}^{-2} \mathrm{~K}^{-1}$ positive longwave feedback compensation.

Soden et al. (2008) showed that the CMIP-3 GCMs display a compensating relationship between the lapse rate feedback and the water vapor feedback. This was explained, assuming constant relative humidity, such that a model with a large negative lapse rate feedback develops greater temperatures aloft, thus allowing the specific humidity to be greater and thus increasing the water vapor feedback (Zhang et al. 1994). This relationship is reproduced in Fig. 3d, and is also shown to hold for the climateprediction.net models.

\subsection{Multi-feedback EOFs}

A fundamental question in the use of perturbed physics ensembles is whether they are able to emulate the range of responses seen in a multi-model ensemble such as CMIP-3. We can examine the first order modes of variation in feedback response in an ensemble using Principal Component Analysis (Sanderson et al. 2008a). Such an analysis yields the dominant patterns of feedback response which scale across the ensemble, so if similar patterns were apparent in both the CMIP-3 and climateprediction.net ensembles, one could conclude that similar physical processes were being sampled by the ensemble. It should be noted that there is some danger in over-interpreting the physical significance of EOFs, as the imposed requirement of orthogonality can introduce artifacts, especially when different modes have similar eigenvalues (North et al. 1982)-hence we examine the significance of only the leading, well separated modes.

EOFs show the dominant spatial modes of variation in feedback response about the ensemble mean, so we first compare the ensemble mean feedback profiles for both ensembles (Fig. 4). Both show similar properties, with compensating negative lapse rate and positive longwave water vapor feedbacks as the dominant features. Both ensemble means show positive longwave cloud feedbacks in equatorial regions. Additionally, the climateprediction.net mean shows a positive net feedback in the northern hemisphere extratropics, while the CMIP-3 mean shows a negative feedback polewards of $60^{\circ} \mathrm{N}$. In the tropics, the climateprediction.net mean profile shows net negative shortwave cloud feedback throughout while the CMIP-3 mean is close to zero at the equator rising to positive values of $1 \mathrm{Wm}^{-2} \mathrm{~K}^{-1}$ at $\pm 20^{\circ}$ North and South.

Figure 4 also shows the first two EOFs in each ensemble. We consider first CMIP-3, where the first EOF accounting for almost $27 \%$ of the variance shows dominant differences between the $\triangle \mathrm{CRF}$ response of the models. Some compensation is clearly visible, with shortwave and longwave feedbacks tending to act in the opposite direction. Shortwave feedback response shows a distinct peak along the Inter-Tropical Convergence Zone (ITCZ) which is partly compensated by an opposing longwave response suggesting a role of high clouds. The uncompensated 
Fig. 4 A comparison of the ensemble mean zonally averaged feedback profiles for both the climateprediction.net (bottom) and the CMIP-3 (top) ensembles. Also shown are the first two multi-variable EOFs generated as described in Sect. 2.4. Values in the top left of each plot represent the percentage variance associated with each mode. Vertical axes for EOFs represent normalized feedback values and are therefore unitless, but relative sizes of different feedbacks are representative of their true relationship
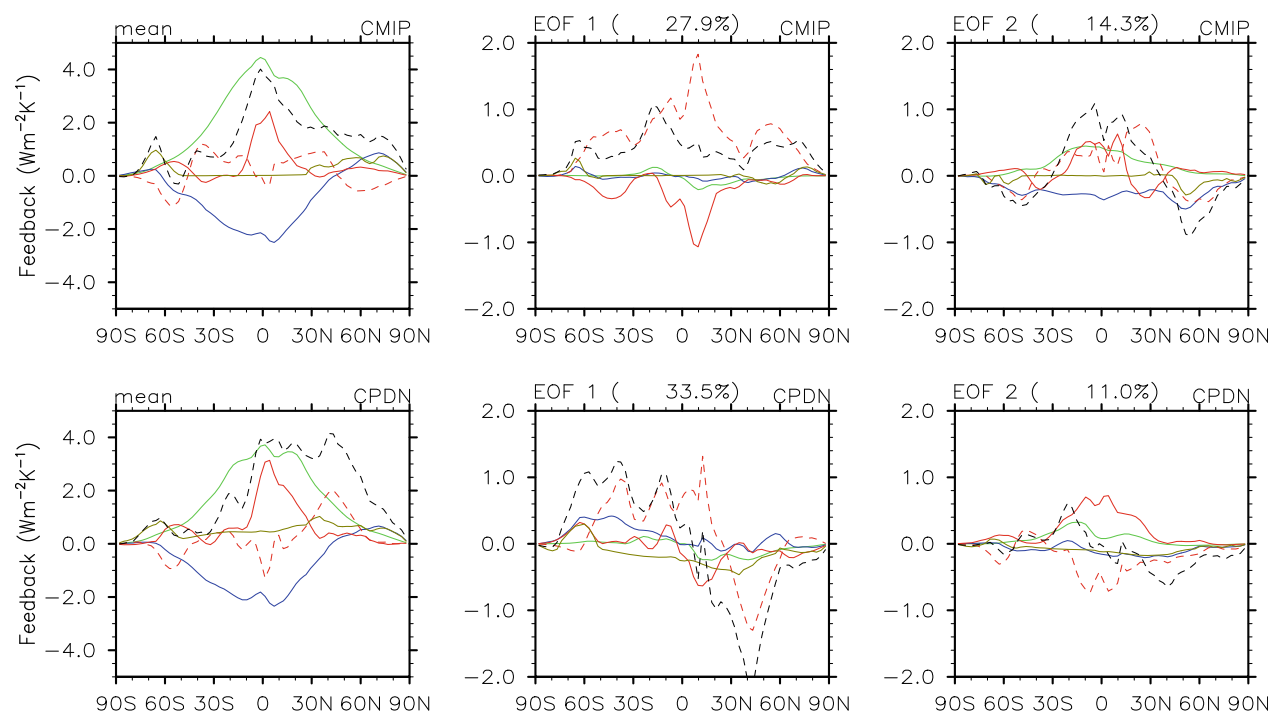

shortwave response, and hence the difference in global mean $\triangle \mathrm{CRF}$, is at higher latitudes and likely due to changes in low cloud which would have little effect on the longwave budget.

Further EOFs in the CMIP-3 ensemble display a more complex interaction of feedback processes. The second EOF, which accounts for $14.2 \%$ of the variance in the CMIP-3 ensemble, shows the established anti-correlation between water vapor feedback and lapse rate feedback that was seen in Fig. 3. However, additional spatial features are now also visible; the LW water vapor feedback shows a smooth, symmetrical latitudinal profile, peaking at the equator and becoming less significant towards the poles. Meanwhile, the lapse rate feedback variation appears constant in the tropics, with a peak in the northern hemisphere mid-latitudes, showing the importance of landmasses and perhaps moisture availability in the amplitude of the lapse rate feedback. Thus, although there is global anticorrelation between the lapse rate and water vapor feedback, the degree of compensation is dependent upon the latitude (Zhang et al. 1994).

It is also apparent that there is some correlated cloud response with the water vapor feedback (although the direct effect of water vapor on $\triangle \mathrm{CRF}$ has been removedwith some assumptions, as described in Sect. 2.1). GCMs showing a greater water vapor feedback than the mean appear to show a complex, but net positive pattern of cloud feedback as one might expect. In the tropics of GCMs with a larger water vapor feedback than the mean, we see a positive longwave and shortwave $\triangle \mathrm{CRF}$ response. Soden and Held (2006) show that most of the spread in lapse rate feedback (and also in water vapor feedback) in the CMIP-3 ensemble arises from differences in the latitudinal distribution of warming, hence it seems likely that models with a strong positive cloud feedback are experiencing greater tropical warming, and thus more negative lapse rate feedback and more positive water vapor feedback due to increased tropospheric moistening. The similar eigenvalues of the higher order modes mean that they cannot be considered unique, hence we do not attempt to attach a physical significance to further EOF patterns.

In the climateprediction.net ensemble, it is immediately apparent that different processes dominate the variation in feedback response. Whereas the first mode in the CMIP-3 ensemble showed longwave-shortwave cloud feedback compensation, the first mode in climateprediction.net accounts for $33 \%$ of the variance and shows mainly variation in the shortwave component of the cloud feedback. Interestingly, there is an anti-correlation between the sign of the response in the Northern Extra-tropics and the rest of the globe, suggesting that there may be parameter changes which have the opposite effects on shortwave cloud feedbacks over land and ocean.

The second EOF shows the expected global compensation between longwave and shortwave cloud feedbacks, but this mode only accounts for $11 \%$ of the total variance. Both the first mode and the second mode show a local variation in lapse rate feedback not compensated by an opposing change in water vapor feedback-which is markedly different behavior to that seen in the CMIP-3 ensemble. 
Further modes (not shown) again become increasingly complex, with similar eigenvalues and are thus difficult to attribute firm physical meaning.

\subsection{Parameter dependency}

In order to understand the physical processes behind the variation of feedback processes in the climateprediction.net ensemble, it is of some use to consider the parameter perturbations themselves. In Fig. 5, we examine the correlations between perturbed parameters in the ensemble and different global mean feedback components as revealed by the kernel method.

It is apparent that a small number of parameters drive most of the variation. The already-mentioned entrainment coefficient again features strongly in Fig. 5, with an increase in value causing positive perturbations in the lapse rate and cloud feedbacks, coupled with a reduction in water vapor feedback as expected from Fig. 3. ENTCOEF also shows the largest correlation with the surface albedo feedback. Figure 3 also showed that the kernel estimates of total clear-sky feedback were underestimated for low values of ENTCOEF, but this is most likely due to an underestimate of the greenhouse gas forcing in these simulations.

Other parameters of importance are revealed from Fig. 5: the accretion constant CT) affects cloud, water vapor and lapse rate feedbacks, the cloud water threshold for precipitation (co-parameters CW_SEA over the ocean and CW_LAND over land) shows compensating water vapor and lapse rate feedbacks; the scaling parameter for ice fall velocity (VF1) affects the cloud feedback, as does the Empirically Adjusted Cloud Fraction (EACF)—which is a direct scaling factor for the degree of cloudiness for a given temperature and humidity. Finally, the critical relative humidity for the formation of clouds (RHcrit) also shows a large correlation with the cloud feedback in the climateprediction.net ensemble.

We can understand each of these correlations better by considering how the EOFs of feedback response project onto the parameter space (Fig. 6). The aim is to estimate the change in zonally averaged feedback response (for each of the kernel types considered) that results from a positive perturbation of each parameter in turn (the amplitude of the perturbation is set by the climateprediction.net experimental design, where expert solicitation was used for each parameter to determine 'reasonable' limits of physical uncertainty). The methodology is discussed in Sect. 2.4.

An increase in the ice fall speed, VF1 causes a strong nearly compensating positive shortwave and negative longwave cloud feedback responses in the tropics, coupled with compensated negative water vapor feedback and positive lapse rate feedback (Fig. 6a). In the Northern extratropics, the negative longwave cloud feedback change is uncompensated by a shortwave change-leading to the net negative cloud feedback perturbation (for a positive perturbation of VF1) seen in Fig. 5. A larger ice fall speed parameter results in a decrease in control simulation cloud area (Wu (2002) and Grabowski (2000) both found that large ice fall speeds in single column models cause a drier, cooler, less cloudy troposphere). This decrease in cloud
Fig. 5 Correlation coefficients between perturbed parameter values in climateprediction.net and various kernel-derived global mean feedbacks

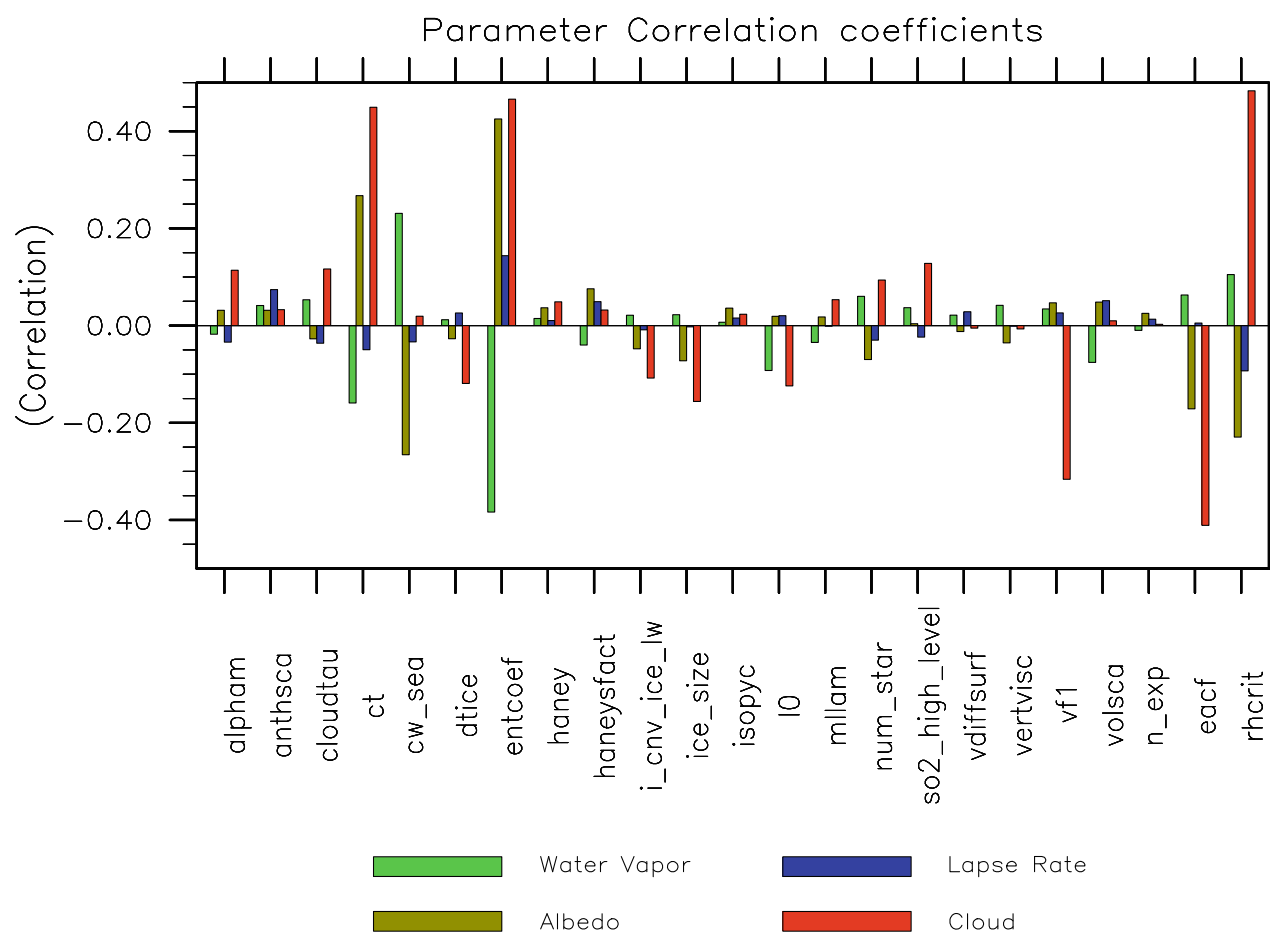


(a)

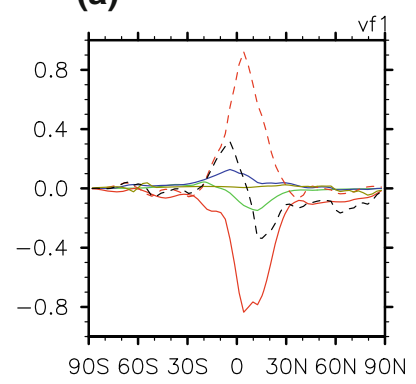

(c)

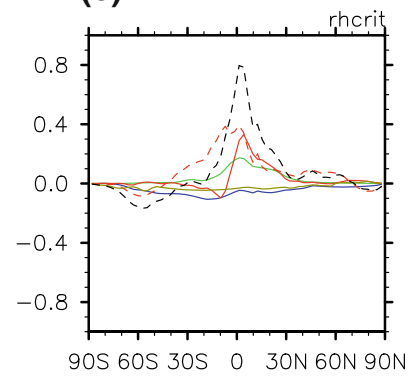

(e)

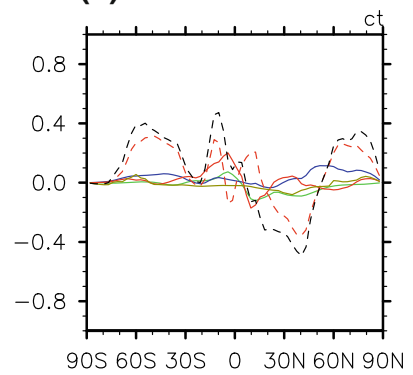

(b)

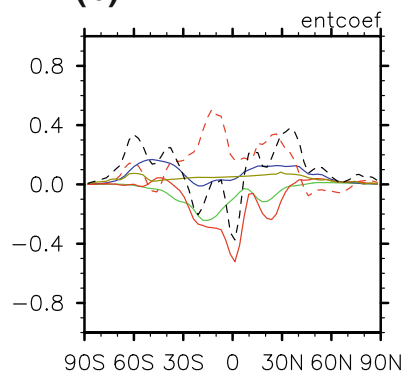

(d)

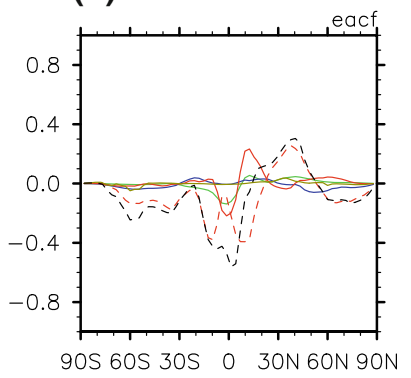

(f)

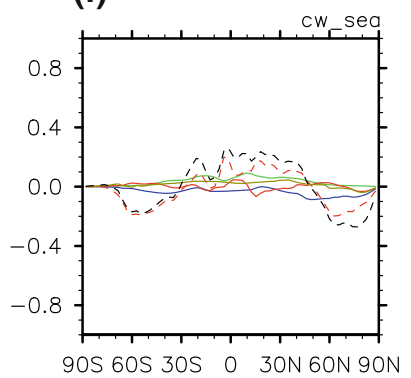

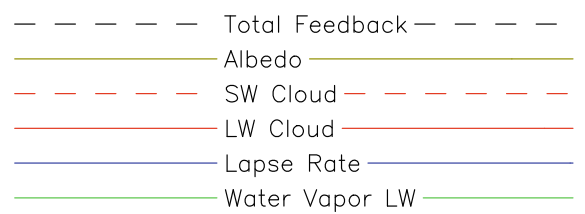

Fig. 6 Multi-variable EOFs of feedback amplitude projected onto single parameters using the methodology described in Sect. 2.4. Feedback patterns may be interpreted as the change from the mean climateprediction.net feedback in $\mathrm{Wm}^{-2} \mathrm{~K}^{-1}$ as a result of a positive perturbation of each perturbed parameter in turn (where the amplitude of the perturbation is determined by the climateprediction.net experimental design)

area results in compensating decreases in shortwave reflectivity and longwave absorption of outgoing longwave radiation (Kiehl and Ramanathan 1990) in the mean state. Our results suggest that the longwave and shortwave cloud feedbacks controlled by VF1 are proportional to the longwave and shortwave cloud forcing present in the control simulation; with less cloud in the mean state, the climateprediction.net mean cloud feedback shown in Fig. 4 is reduced to give the perturbation signal shown in Fig. 6 (but the effect is noticeably concentrated around the ITCZ).
An increase in the entrainment coefficient, ENTCOEF, also causes compensating cloud feedbacks (positive shortwave and negative longwave) in the tropics (Fig. 6b). However, this time the tropical humidity feedback is uncompensated by a lapse rate feedback. This suggests that the increased humidity feedback is not due to increased temperatures aloft and constant relative humidity (as was the case for VF1), but that the ENTCOEF perturbation alters the relative humidity distribution in the tropics. Sanderson et al. (2008b) found that an effect of an decrease in ENTCOEF was to moisten the upper troposphere/lower stratosphere region while drying the lower troposphere in the tropics.

In order to further understand the effect this altered humidity distribution might cause on clear sky feedbacks, it is useful to study the kernel derived field effect on model levels. The field effect is the product of the kernel with the $\Delta \mathrm{T}$ field in the model, divided by the global mean $\Delta \mathrm{T}$. Hence when integrated over the vertical, it should represent the portion of the total feedback due to changes in temperature alone (which was shown in Fig. 6). Figure 7c shows the perturbation in the kernel derived field effect which is observed upon a positive perturbation of ENTCOEF in the model. From this plot, we can infer that a positive perturbation of ENTCOEF produces a model which, when subjected to $\mathrm{CO}_{2}$ forcing, creates a more negative atmospheric temperature feedback in the boundary layer (i.e. the model's boundary layer warms more than average for a given increase in surface temperature). However, there is also a compensating more positive atmospheric temperature feedback in the tropical mid-troposphere (i.e. there is less warming than average at altitude for a given increase in surface temperature, resulting in a weaker (negative) lapse rate feedback for a positive perturbation of ENTCOEF). Comparing back to Fig. 6, this compensation (between upper and lower atmosphere effects) is complete in the tropics but not in the extratropics where the lower atmosphere negative feedback dominates.

The water vapor field effect corresponding to a positive ENTCOEF perturbation weakly compensates the temperature field effect (Fig. 7a) — the two effects are not strongly compensating because the tropical troposphere is moistened significantly with a increase of ENTCOEF (see Sanderson et al. 2008b, Fig. 5). However, Fig. 6b shows that the net water vapor feedback in the tropics with a positive perturbation of ENTCOEF is weakened. Figure 7a suggests that this is due to decreased upper tropospheric moistening.

A surprising aspect of these results is the apparent nearcancellation of the feedback changes resulting in a perturbation of ENTCOEF when integrated globally, given the findings of previous studies (Stainforth et al. 2005; Sanderson et al. 2008b; Sanderson et al. 2008a) which suggested the dominant influence of the parameter in determining climate sensitivity. There are various 

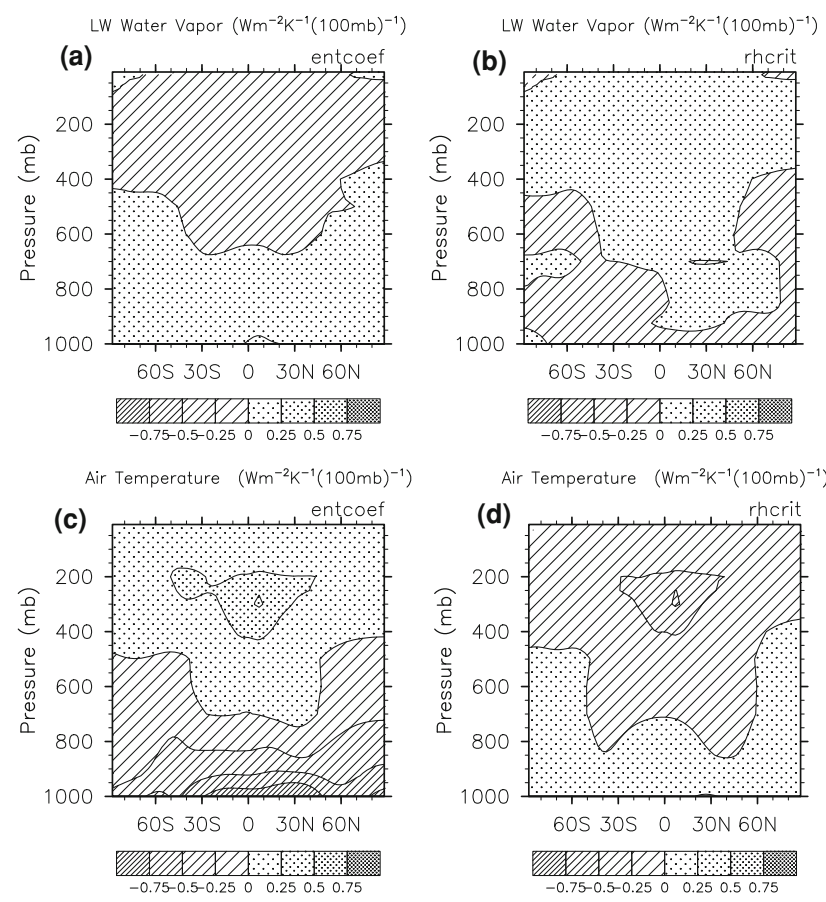

Fig. 7 Field effect perturbations corresponding to positive parameter changes, resolved on model levels. Field effects are the product of the radiative kernel with the atmospheric changes in the model, divided by the global mean temperature increase. Contours show $\mathrm{Wm}^{-2} \mathrm{~K}^{-1}(100 \mathrm{mb})^{-1}$ such that integrating over the vertical levels yields the zonal feedback plots shown in Fig. 6

explanations for this behavior; firstly Sanderson et al. (2008b) showed that in order to simulate very high sensitivities in the HadAM3 model it was necessary to perturb multiple parameters simultaneously. This behavior will not be apparent from the linear analysis shown in Figs. 6 and 7. Secondly, as discussed in Sect. 3.3, there is a consistent underestimation of the true feedback strength in models with a reduced value for ENTCOEF, which is most likely attributable to an underestimation of the true greenhouse gas forcing in these models.

The effects of an increase in the critical relative humidity for cloud formation, RHcrit, (Smith 1990) are positive increases in longwave and shortwave cloud feedback (Fig. 6c). The lack of compensation, together with the differing zonal structure for the shortwave and longwave cloud feedback, suggest that the parameter is having differing effects on boundary layer clouds and high level clouds. This is consistent with the findings of Pope et al. (2000), who found that a reduction of RHcrit led to an increase in the amount of water cloud and a decrease in the amount of ice cloud. The shortwave feedback is positive, and symmetric about the equator. One would expect a reduction in boundary layer cloud with an increase in the minimum relative humidity required for cloud formation. Again, we might expect that the low-level cloud feedback would scale with the low-level cloud amount. Figure 4 shows that the climateprediction.net mean shortwave cloud feedback in the tropics is negative, and thus we speculate that a reduction in the control low level cloud amount might reduce the amplitude of the negative shortwave cloud feedback when globally averaged, resulting in the positive perturbation on the mean response shown in Fig. 6c.

Changes in boundary layer clouds would have little effect on the longwave budget, so we infer that the positive longwave cloud feedback in the ITCZ is due to an increase in high level clouds. The increase in available moisture aloft allows greater high cloud cover-despite the increased relative humidity required for the clouds to form. By examining the field effect on model levels in Fig. $7 \mathrm{~b}$ and $\mathrm{d}$ ), we see that an increase in RHcrit produces a stronger water vapor feedback than the default model, especially in the tropics (this is more than compensated by a more negative lapse rate feedback).

The remaining parameters in Fig. 6 have more complex feedback signatures-both the empirically adjusted cloud fraction EACF and the accretion constant CT show similar (but opposite) zonal patterns of shortwave cloud feedback (with some longwave compensation). The changes are a direct scaling of boundary layer cloud amount, enhancing the changes in cloud fraction which would otherwise occur (not shown). The parameters show little to no shortwave cloud effect at the equator, but large peaks at $\pm 15^{\circ}$ and polewards of $\pm 60^{\circ}$. This suggests that the parameters are influencing mainly shortwave cloud feedbacks in regions of zonally averaged subsidence, which would suggest that marine boundary layer cloud processes of the type discussed by Bony and Dufresne (2005) are being modulated. Furthermore, between 30 and $60^{\circ} \mathrm{N}$, the change in shortwave feedback is of the opposite direction-suggesting that the parameters are having the opposite effect over the Northern Hemisphere landmass (and perhaps land in general).

These changes can be understood physically in terms of the parameters: an increase in the empirically adjusted cloud fraction is a direct scaling up of the cloud amount for a given temperature and humidity, thus any increase in boundary layer cloud will be amplified over the oceans, as will any decrease over the landmass. Similarly a decrease in the accretion constant reduces the rate at which cloud water is accreted onto falling precipitation, thus having a similar effect of maintaining a larger cloud fraction for given conditions and amplifying any changes in boundary layer cloud distribution on warming.

Finally, the water content thresholds for precipitation over sea and land, CW_SEA and CW_LAND, show a large influence over shortwave cloud, water vapor and lapse rate feedbacks. Globally, an increase in the threshold causes a positive humidity feedback, as larger humidities persist without initiating precipitation. In the tropics, an increase in the threshold causes an increase in shortwave 
cloud feedback while at higher latitudes it causes a decrease. The effect on lapse rate feedback is negative between 40 and $60^{\circ} \mathrm{N}$, but minimal elsewhere.

\section{Summary}

In this paper, we have applied the radiative kernel technique developed by Soden and Held (2006) to a selection of transient simulations from the perturbed physics ensemble, climateprediction.net. Radiative kernels allow the top of atmosphere response in a climate change simulation to be decomposed into a sum of feedbacks resulting from changes in various independent atmospheric variables. The climate feedback is the product of two factors, a kernel which is diagnosed by perturbing the base climate variable by a small amount and observing the top of atmosphere radiative response, and the corresponding variable taken from a climate change simulation. We use radiative kernels developed using the NCAR Community Atmosphere Model by Shell et al. (2008) to identify feedbacks due to changes in water vapor, atmospheric temperature, surface albedo and temperature. Estimates for the longwave and shortwave cloud radiative forcing response include corrections to exclude the effects of changing atmospheric transmission as described in Soden et al. (2008).

Several obstacles prevented an identical treatment of the data to that used in Soden et al. (2008); atmospheric variables are zonally averaged in climateprediction.net, forcing the use of zonal mean kernels to approximate the response. To test the legitimacy of this approach, we repeated the experiments conducted by Soden et al. (2008) on the CMIP-3 ensemble, comparing the global mean feedback responses yielded by both zonally averaged and fully gridded kernels. The zonally averaged kernels are an adequate approximation for the full kernels, with typical errors of less than $0.1 \mathrm{Wm}^{-2} \mathrm{~K}^{-1}$. A lack of surface diagnostics in climateprediction.net also necessitated some approximation of the surface feedbacks, with surface albedo calculated as a residual and surface temperature assumed to be equal to the unperturbed model.

A verification using longwave clear-sky feedback response shows that the kernel estimate in both climateprediction.net and CMIP-3 is a reasonable approximation for the true, GCM derived radiative feedback response to surface warming. However, in some cases where the entrainment parametrization in the GCM convection scheme has been perturbed, there are significant biases in the zonally averaged relative humidity distribution between the perturbed model and the model used to derive the kernel. The kernel technique underestimates the longwave clear-sky feedbacks in these models, because of errors in the kernelderived estimate of radiative forcing or the feedback itself.
Net cloud feedback was approximated by calculating the change in cloud radiative forcing $(\triangle \mathrm{CRF})$, adjusted to remove the effects of other atmospheric changes. This adjustment is made possible through the use of both clear sky and cloudy sky kernels to measure the component of $\triangle \mathrm{CRF}$ due to changing temperature, humidity or carbon dioxide. Both climateprediction.net and CMIP-3 show a similar range of net cloud feedback, but the mean of the distribution in climateprediction.net is shifted towards more positive values. Thus, although three models in the CMIP-3 ensemble show negative net corrected $\triangle \mathrm{CRF}$ response to surface warming, there are no such models in the climateprediction.net ensemble.

An examination of the leading order EOFs of zonal mean feedback strength across both ensembles reveals this particular perturbed physics ensemble and multi-model ensembles such as CMIP-3 produce very different first order variation in regional feedback strength (it should be noted, however, that this first order variation is fundamentally dependent the arbitrary sampling of parameters used to create the perturbed physics ensemble). Whereas in CMIP-3, the variation is dominated by compensating global scale longwave and shortwave cloud feedbacks, the first mode in the climateprediction.net ensemble shows variation primarily in the shortwave cloud feedback with the opposite sign over the northern hemisphere landmass. This lack of longwave compensation in the first mode provides a plausible explanation for the larger range of climate sensitivities observed in the climateprediction.net ensemble (Stainforth et al. 2005).

Further study of the effects of GCM parameters perturbed in the climateprediction.net experiment shows that the parameters dominating the variation of atmospheric feedbacks are predominantly those identified by Sanderson et al. (2008b) in the original mixed layer ensemble. A projection of feedback patterns onto the parameter space suggests differences in the mechanisms of feedback perturbation that can be observed in the ensemble. Some parameters, such as those scaling the ice fall speed and convective entrainment in the GCM, cause large but compensating changes in shortwave and longwave tropical cloud feedback. We find that the humidity feedback may be altered by perturbing either the entrainment coefficient or the critical relative humidity for cloud formation. Furthermore, we find that although globally, the compensating relationship between water vapor feedback and lapse rate feedback remains robust in a perturbed parameter regime, it is not necessarily true locally.

We observe several parameters which appear to directly modulate the shortwave cloud feedback due to low level clouds, including the parameters controlling the accretion of cloud water on precipitation and those directly scaling the cloud fraction. Given that these clouds have been 
identified as a major source of uncertainty in current estimates of global cloud feedback (Bony and Dufresne 2005), further study on the effects and uncertainties associated with these parameters is necessary.

Acknowledgments Thanks to Brian Soden and an anonymous reviewer for their detailed input. This work was only made possible with the efforts of the team who developed and continue to support the climateprediction.net project, as well as the members of the public who donated their idle computing time to the project. We acknowledge the modeling groups, the Program for Climate Model Diagnosis and Inter-comparison (PCMDI) and the WCRP's Working Group on Coupled Modeling (WGCM) for their roles in making available the WCRP CMIP3 multi-model dataset. Support of this dataset is provided by the Office of Science, U.S. Department of Energy. William Ingram was supported by the Joint DECC, Defra and MoD Integrated Climate Programme—DECC/Defra (GA01101), MoD (CBC/2B/0417 Annex C5). This research was supported by the Office of Science (BER), US Department of Energy, Cooperative Agreement No. DEFC02-97ER62402. The National Center for Atmospheric Research is sponsored by the National Science Foundation.

\section{Appendix A: Model setup}

Model simulations are run from 1850 until 2060, using observed $\mathrm{CO}_{2}$ emissions from 1850 until 2000 (Ramaswamy et al. 2001) and the Special Report on Emissions Scenarios' A1B scenario (Nakicenovic et al. 2000) for greenhouse gas emissions between the years 2000 and 2060. Past solar variability assumes one of four possible scenarios: Solanki and Krivova (2003), Hoyt and Schatten (1993), Lean et al. (1995) or Lockwood (2002). For future solar forcing, three scenarios are considered for each past reconstruction, resulting in twelve 1820-2060 solar forcing scenarios. The future solar scenarios repeat the corresponding past solar forcing, with a trend which is zero, equal to the 1920-2000 trend or the negative of the 1920-2000 trend. The model also includes a sulfur cycle and is sensitive to aerosols emitted by volcanic eruptions. Past volcanic aerosol reconstructions are provided by Ammann et al. (2003) or Sato et al. (1993) (updated to 2002). There are ten possible future volcanic aerosol scenarios, one scenario simply repeats the recent past according to the updated Sato et al. (1993) data set. Two more are based on observations of the preceding 80 years, based on the Sato et al. (1993) and Ammann et al. (2003) data sets. The remaining 7 are subsets of observations of 1400-1960, based on a data set constructed by Crowley (2000).

\section{References}

Ammann C, Meehl G, Washington W, Zender C (2003) A monthly and latitudinally varying volcanic forcing dataset in simulations of 20th century climate. Geophys Res Lett 30(12):1657
Bony S and Dufresne J-L (2005) Marine boundary layer clouds at the heart of tropical cloud feedback uncertainties in climate models. Geophys Res Lett 32(20):L20806

Bony S, Colman R, Kattsov VM, Allan RP, Bretherton CS, Dufresne J-L, Hall A, Hallegatte S, Holland MM, Ingram W, Randall DA, Soden BJ, Tselioudis G, and Webb MJ (2006) How well do we understand and evaluate climate change feedback processes? J Clim 19(15):3445-3482

Caldeira K, Jain A, and Hoffert M (2003) Climate sensitivity uncertainty and the need for energy without $\mathrm{CO}_{2}$ emission. Science 299(5615):2052-2054

Cess RD, Potter G (1988) A methodology for understanding and intercomparing atmospheric climate feedback processes in general-circulation models. J Geophys Res Atmos 93(D7): 8305-8314

Collins W, Ramaswamy V, Schwarzkopf M, Sun Y, Portmann R, Fu Q, Casanova S, Dufresne J, Fillmore D, Forster P et al. (2006) Radiative forcing by well-mixed greenhouse gases: estimates from climate models in the IPCC AR4. J Geophys Res 111(D14):D14317

Colman R (2003) A comparison of climate feedbacks in general circulation models. Clim Dynam 20(7-8):865-873

Colman R, Power S, McAvaney B (1997) Non-linear climate feedback analysis in an atmospheric general circulation model. Clim Dynam 13(10):717-731

Crowley TJ (2000) Causes of climate change over the past 1000 years. Science 289(5477):270-277

Forster P, Gregory J (2006) The climate sensitivity and its components diagnosed from earth radiation budget data. J Clim 19(1):39-52

Grabowski W (2000) Cloud microphysics and the tropical climate: cloud-resolving model perspective. J Clim 13(13):2306-2322

Hall A and Qu X (2006) Using the current seasonal cycle to constrain snow albedo feedback in future climate change. Geophys Res Lett 33(3):L03502

Hansen J, Russell G, Lacis A, Fung I,Rind D (1985) Climate response times: dependence on climate sensitivity and ocean mixing. Science 229:857-859

Held IM, Soden BJ (2000) Water vapor feedback and global warming. Annu Rev Energ Env 25:441-475

Hoyt D, Schatten K (1993) A discussion of plausible solar irradiance variations, 1700-1992. J Geophys Res 98(A11): 18895-18906

Kiehl J, Ramanathan V (1990) Comparison of cloud forcing derived from the earth radiation budget experiment with that simulated by the NCAR community climate model. J Geophys Res Atmos 95(D8):11679-11698

Knutti R, Allen MR, Friedlingstein P, Gregory J, Hegerl G, Meehl GA, Meinshausen M, Murphy JM, Plattner G-K, Raper S, Stott PA, Teng H, and Wigley TML (2008) A review of uncertainties in global temperature projections over the twenty-first century. J Clim 21:2651-2663

Lean J, Beer J, Bradley R (1995) Reconstruction of solar irradiance since 1610-implications for climate-change. Geophys Res Lett 22(23):3195-3198

Lockwood M (2002) An evaluation of the correlation between open solar flux and total solar irradiance. Astron Astrophys 382(2):678-687

Murphy JM, Sexton DMH, Barnett D, Jones G, Webb MJ,Collins M (2004) Quantification of modelling uncertainties in a large ensemble of climate change simulations. Nature 430(7001):768772

Murphy JM, Booth BBB, Collins M, Harris GR, Sexton DMH, Webb MJ (2007) A methodology for probabilistic predictions of regional climate change from perturbed physics ensembles. Philos T R Soc A 365(1857):1993-2028 
Nakicenovic N, Alcamo J, Davis G, de Vries B, Fenhann J, Gaffin S, Gregory K, Grubler A, Jung T, Kram T et al (2000) Special report on emissions scenarios: a special report of Working Group III of the Intergovernmental Panel on Climate Change, PNNLSA-39650. Cambridge University Press, New York, NY (US)

North GR, Bell T, Cahalan R, Moeng F (1982) Sampling errors in the estimation of empirical orthogonal functions. Mon Weather Rev 110(7):699-706

Pope V, Gallani M, Rowntree P, Stratton R (2000) The impact of new physical parametrizations in the hadley centre climate model: Hadam3. Clim Dynam 16(2-3):123-146

Ramaswamy V, Boucher O, Haigh J, Hauglustaine D, Haywood J, Myhre G, Nakajima T, Shi G, Solomon S, Betts R et al (2001). In: Houghton JT et al (eds) Radiative forcing of climate change, PNNL-SA-39648, Cambridge University Press, New York

Sanderson BM, Knutti R, Aina T, Christensen C, Faull N, Frame DJ, Ingram W, Piani C, Stainforth DA, Stone DA, Allen MR (2008a) Constraints on model response to greenhouse gas forcing and the role of subgrid-scale processes. J Clime 21(11):2384-2400

Sanderson BM, Piani C, Ingram W, Stone DA, Allen MR (2008b) Towards constraining climate sensitivity by linear analysis of feedback patterns in thousands of perturbed-physics GCM simulations. Clim Dynam 30(2-3):175-190

Sato M, Hansen J, McCormick M, Pollack J (1993) Stratospheric aerosol optical depths, 1850-1990. J Geophys Res 98(D12): 22987-22994

Shell KM, Kiehl JT, Shields CA (2008) Using the radiative kernel technique to calculate climate feedbacks in NCAR's community atmospheric model. J Clim 21(10):2269-2282

Smith R (1990) A scheme for predicting layer clouds and their watercontent in a general-circulation model. Q J Roy Meteorol Soc 116(492):435-460

Soden BJ, Broccoli A, Hemler R (2004) On the use of cloud forcing to estimate cloud feedback. J Clim 17(19):3661-3665
Soden BJ, Held IM (2006) An assessment of climate feedbacks in coupled ocean-atmosphere models. J Clim 19(14):3354-3360

Soden BJ, Held IM, Colman R, Shell KM, Kiehl JT, Shields CA (2008) Quantifying climate feedbacks using radiative kernels. J Clim 21(14):3504

Solanki S, Krivova N (2003) Can solar variability explain global warming since 1970? J Geophys Res 108(A5):1200

Stainforth DA, Aina T, Christensen C, Collins M, Faull N, Frame DJ, Kettleborough JA, Knight SHE, Martin A, Murphy JM, Piani C, Sexton DMH, Smith L, Spicer R, Thorpe A, Allen MR (2005) Uncertainty in predictions of the climate response to rising levels of greenhouse gases. Nature 433(7024):403-406

Tebaldi C, Knutti R (2007) The use of the multi-model ensemble in probabilistic climate projections. Philos T R Soc A 365(1857): 2053-2075

Webb MJ, Senior C, Sexton DMH, Ingram W, Williams KD, Ringer MA, McAvaney B, Colman R, Soden BJ, Gudgel R, Knutson T, Emori S, Ogura T, Tsushima Y, Andronova N, Li B, Musat I, Bony S, and Taylor KE (2006) On the contribution of local feedback mechanisms to the range of climate sensitivity in two GCM ensembles. Clim Dynam 27(1):17-38

Wetherald R, Manabe S (1988) Cloud feedback processes in a general-circulation model. J Atmos Sci 45(8):1397-1415

Wu X (2002) Effects of ice microphysics on tropical radiativeconvective-oceanic quasi-equilibrium states. J Atmos Sci 59(11):1885-1897

Yokohata T, Emori S, Nozawa T, Ogura T, Kawamiya M, Tsushima Y, Suzuki T, Yukimoto S, A. Abe-Ouchi, Hasumi H, Sumi A, Kimoto M (2008) Comparison of equilibrium and transient responses to $\mathrm{CO}_{2}$ increase in eight state-of-the-art climate models. Tellus A 60(5):946-961

Zhang M, Hack JJ, Kiehl JT, Cess RD (1994) Diagnostic study of climate feedback processes in atmospheric general circulation models. J Geophys Res 99(D3):5525-5537 